For submission to Biochemical Pharmacology

\title{
Cloning and activity of a novel \\ $\alpha$-latrotoxin from red-back spider venom ${ }^{\#}$
}

\author{
Andis Graudins ${ }^{a, \dagger}$, Michelle J. Little ${ }^{a}$, Sandy S. Pineda ${ }^{b}$, Peter G. Hains ${ }^{c, \ddagger}$, Glenn F. King ${ }^{b}$, \\ Kevin W. Broady ${ }^{a}$, Graham M. Nicholson*a \\ a Neurotoxin Research Group, School of Medical \& Molecular Biosciences, University of Technology, Sydney, Broadway NSW 2007, Australia \\ Institute for Molecular Bioscience, University of Queensland, St. Lucia, QLD 4072 Australia \\ Save Sight Institute, Sydney Eye Hospital, Macquarie Street, Sydney NSW 2001, Australia
}

Key words: Latrodectus, latrotoxin, $\alpha$-LTX-Lh1a, latroinsectotoxin, neurotransmitter release

\# Ethical statement: The authors declare that all animal experiments described in the paper comply with Australian animal ethics regulations.

* Corresponding author. Professor Graham Nicholson, PhD

Neurotoxin Research Group

School of Medical \& Molecular Biosciences

University of Technology, Sydney

PO Box 123

Broadway, NSW, 2007

Australia

Tel.: +61-2-9514-2230; fax: +61-2-9514-8206

E-mail address: Graham.Nicholson@uts.edu.au (G. Nicholson). 
$\dagger \quad$ Present address: Southern Health Emergency Medicine Research Group, Southern Clinical School, Faculty of Medicine, Nursing \& Health Sciences, Monash University, Clayton, VIC 3168, Australia

$\ddagger \quad$ Present address: Cell Signaling Unit, Children's Medical Research Institute, Westmead, NSW 2145, Australia

The DNA sequence of $\alpha$-latrotoxin-Lh1a ( $\alpha$-LTX-Lh1a) reported in this paper has been deposited in the EMBL Nucleotide Sequence Database, under Accession Number FR851877, and the ArachnoServer Spider Toxin Database (ID\#1290). The deduced amino acid sequence of $\alpha$-LTXLh1a has also been deposited in the ArachnoServer Spider Toxin Database. 


\section{ABSTRACT}

The venom of the European black widow spider Latrodectus tredecimguttatus (Theridiidae) contains several high molecular mass $(110-140 \mathrm{kDa})$ neurotoxins that induce neurotransmitter exocytosis. These include a vertebrate-specific $\alpha$-latrotoxin ( $\alpha$-LTX-Lt1a) responsible for the clinical symptoms of latrodectism and numerous insect-specific latroinsectoxins (LITs). In contrast, little is known about the expression of these toxins in other Latrodectus species despite the fact that envenomation by these spiders induces a similar clinical syndrome. Here we report highly conserved $\alpha$-LTX, $\alpha$-LIT and $\delta$-LIT sequence tags in L. mactans, $L$. hesperus and $L$. hasselti venoms using tandem mass spectrometry, following bioassay-guided separation of venoms by liquid chromatography. Despite this sequence similarity, we show that the anti- $\alpha$-LTX monoclonal antibody $4 C 4.1$, raised against $\alpha-L T X-L t 1 a$, fails to neutralize the neurotoxicity of all other Latrodectus venoms tested in an isolated chick biventer cervicis nerve-muscle bioassay. This suggests that there are important structural differences between $\alpha-L T X s$ in theridiid spider venoms. We therefore cloned and sequenced the $\alpha$-LTX from the Australian red-back spider L. hasselti $(\alpha-$ LTX-Lh1a). The deduced amino acid sequence of the mature $\alpha$-LTX-Lh1a comprises 1180 residues $(\sim 132 \mathrm{kDa})$ with $\sim 93 \%$ sequence identity with $\alpha$-LTX-Lt1a. $\alpha$-LTX-Lh1a is composed of an $\mathrm{N}$-terminal domain and a central region containing 22 ankyrin-like repeats. The presence of two furin cleavage sites, conserved with $\alpha$-LTX-Lt1a, indicates that $\alpha$-LTX-Lh1a is derived from the proteolytic cleavage of an $\mathrm{N}$-terminal signal peptide and $\mathrm{C}$-terminal propeptide region. However, we show that $\alpha$-LTX-Lh1a has key substitutions in the 4C4.1 epitope that explains the lack of binding of the monoclonal antibody.

Abbreviations: $\alpha$-LTX, $\alpha$-latrotoxin; BSA, bovine serum albumin; 4C4.1, murine monoclonal antibody raised against $\alpha$-latrotoxin from $L$. tredecimguttatus; CBCNM, chick biventer cervicis nerve-muscle; CNS, central nervous system; ESI-Q-TOF, electrospray ionization quadrupole timeof-flight; FPLC, fast performance liquid chromatography; gDNA, genomic DNA; HEPES, N-2hydroxyethylpiperazine- $N$-2-ethanesulfonic acid; $\alpha$-LCT, $\alpha$-latrocrustatoxin; $L_{50}$, median lethal dose; LIT, latroinsectotoxin; LMWP, low molecular weight protein (also known as latrodectin); MSMS, tandem mass spectrometry; RP-HPLC, reverse-phase high-performance liquid chromatography; SDS-PAGE, sodium dodecyl sulphate polyacrylamide gel electrophoresis; TFA, trifluoroacetic acid. 


\section{INTRODUCTION}

The most clinically significant group of spiders worldwide are the widow or comb-footed spiders, belonging to the genus Latrodectus (Araneae: Araneomorphae: Theridiidae). Widow spider envenomation manifests clinically as latrodectism, resulting in pain either at the bite site or radiating proximally to the whole of the bitten limb, local and regional diaphoresis, and less commonly piloerection and muscle fasciculation. Symptoms may become severe and generalized and include hypertension, tachycardia, nausea, vomiting, and abdomen, back or chest pain. Although death is uncommon, patients may be left with symptoms of envenomation for days or weeks if left untreated [1, 2].

The toxin responsible for the clinical symptoms of latrodectism is the vertebrate-specific $\alpha$-latrotoxin $(\alpha-L T X)$ which has been isolated from the venom of the European widow spider, L. tredecimguttatus [3]. $\alpha-\mathrm{LTX}$ is a large $\sim 130 \mathrm{kDa}$ hydrophilic protein that elicits massive spontaneous neurotransmitter release from neuronal and neuroendocrine nerve terminals [4-6]. Under non-reducing conditions, $\alpha$-LTX exists as a homodimer [7] with each monomer composed of four domains: a signal peptide (domain I), a conserved N-terminal domain with two hydrophobic segments that has no substantial homology to known proteins (domain II), a domain composed of 20 ankyrin repeats (or 22 if two imperfect repeats are included; domain III), and a C-terminal propeptide domain (domain IV) [8]. $\alpha$-LTX is synthesized as a larger preprotoxin of $\sim 157 \mathrm{kDa}$ where domains I and IV are believed to be removed by proteolytic cleavage during venom maturation by the endopeptidase furin, leaving a mature $130 \mathrm{kDa}$ toxin composed of domains II and III [8-11]. Using electron cryo-microscopy, the 3D structure of the mature $\alpha-L T X$ monomer was found to contain three regions: the wing (majority of domain II), the body (one quarter of domain II and first 15-16 ankyrin repeats) and the head (ca. 4.5 C-terminal ankyrin repeats) [7].

L. tredecimguttatus venom also contains five insect-specific toxins known as latroinsectotoxins (LIT) $\alpha, \beta, \gamma, \delta$ and $\varepsilon$ [12], and one crustacean-specific protein, $\alpha$-latrocrustatoxin ( $\alpha$-LCT) [13-15]. Of these, an additional three latrotoxins have been cloned and fully sequenced: $\alpha$-LCT [16], $\alpha$-LIT [9] and $\delta$-LIT [10] with molecular masses of 110-140 kDa. In addition, two acidic low molecular weight proteins (LMWPs or latrodectins; $\sim 7.9 \mathrm{kDa}$ ) have been found to co-purify with $\alpha$-LTX [1719]. $\alpha$-LTX-associated LMWPs are structurally related to crustacean hyperglycemic hormones and are non-toxic $[17,18,20]$. Nevertheless they appear to augment the neurotoxicity of latrotoxins [12, 20], probably by increasing their affinity for the membrane target [21] and reducing vertebrate phyla-specificity such that $\alpha$-LTX becomes active in insects [20].

Currently, $\alpha$-LTX-induced neurotransmitter exocytosis is believed to involve an initial step of $\alpha$-LTX binding to extracellular cell surface membrane proteins. Three structurally-unrelated cell adhesion receptor classes have been identified to mediate this increase in neurotransmitter release by 
$\alpha$-LTX: (i) neurexin $1 \alpha$, a neuronal protein with a single transmembrane domain [22, 23], (ii) latrophilin 1 (CL1 or lectomedin) otherwise known as calcium-independent receptor of $\alpha$-LTX (CIRL; [24, 25]; a member of the CL family of G-protein-coupled receptors [26, 27]), and more recently (iii) receptor-like protein tyrosine phosphatase $\sigma$ [28]. It is believed that these receptors serve as sites to target $\alpha$-LTX to the appropriate location on the cell surface, such as the active zone of nerve terminals at the neuromuscular junction [29]. Once bound, $\alpha-L T X$ oligomerization occurs allowing the formation of amphipathic cyclical tetramers, followed by membrane insertion and non-selective cation channel formation [7]. This pore formation results in an osmotic-mediated increase in vesicular exocytosis and non-vesicular leakage of neurotransmitters. In parallel, vesicle exocytosis ensues as a result of $\mathrm{Ca}^{2+}$ influx through the toxin-induced pore, and receptor-mediated vesicle exocytosis. Interestingly, the anti- $\alpha-\mathrm{LTX}$ monoclonal antibody $4 \mathrm{C} 4.1$ blocks neurotransmitter release by preventing tetramerization of $L$. tredecimguttatus $\alpha-L T X$ and also inhibits the ionophore activity of $\alpha$-LTX once inserted into cell membranes [30,31]. The epitope of the monoclonal antibody $4 \mathrm{C} 4.1$ is located in a hinge region between the body and head of $\alpha-L T X$, thus highlighting the importance of this region in pore formation and toxicity.

While extensive investigations have been carried out on toxins from $L$. tredecimguttatus venom, remarkably little research has been undertaken on the composition of venoms from other Latrodectus species, including the clinically significant Australian red-back spider $L$. hasselti. Nevertheless, the venoms of all Latrodectus species are believed to contain similar $\alpha$-LTX-like proteins since envenomation by any widow spider results in a similar clinical syndrome. Moreover, widow-spider antivenoms, produced using the venoms of specific Latrodectus species, reverse the effects of envenomation from other Latrodectus and Steatoda spiders (another member of the family Theridiidae) both in experimental animal models and in clinical settings [32-35]. The presence of a $130 \mathrm{kDa}$ band in the venom of many theridiid spiders, detected by immunoblotting with red-back spider antivenom, suggests the presence of $\alpha$-LTX-like or antigenically similar proteins in the venoms of all Latrodectus species as well as the related theridiid spider Steatoda grossa [32]. It has therefore been assumed, but not proven, that $\alpha$-LTX is present in the venom of all widow spiders. The aim of the present study was therefore to determine whether the clinical symptoms of envenomation by other Latrodectus species and S. grossa can be explained by the presence of $\alpha$-LTX molecules related to those found in L. tredecimguttatus venom.

\section{MATERIALS AND METHODS}

\subsection{Sources of venoms, toxins and antibodies}

Purified $\alpha$-LTX, derived from $L$. tredecimguttatus, was obtained from Alomone Laboratories (Jerusalem, Israel), reconstituted in $50 \% \mathrm{v} / \mathrm{v}$ glycerol and stored at $-20^{\circ} \mathrm{C}$. Female L. mactans (Southern black widow spider), L. hesperus (Western black widow spider) and L. lugubris (lli River 
Valley widow spider, Kazakhstan) venoms were obtained as lyophilized milked venom, extracted by electrostimulation of the venom apparatus, from Spiderpharm. Female L. tredecimguttatus venom was purchased as lyophilized venom gland extract from Latoxan (Rosans, France). Whilst originally described as a separate theridiid spider species, L. lugubris may simply represent a geographic variant of $L$. tredecimguttatus as Platnick [36] no longer recognizes this species as distinct from $L$. tredecimguttatus. All venoms were reconstituted in water and stored at $-20^{\circ} \mathrm{C}$.

Female L. hasselti (Australian red-back spider) venom was purchased from Venom Supplies Pty. Ltd. (Tanunda SA, Australia), as lyophilized whole venom glands or obtained from venom glands freshly dissected from spiders donated to the Neurotoxin Research Group. Spiders were anaesthetized using $\mathrm{CO}_{2}$. Venom glands were removed through an incision on the dorsum of the cephalothorax and placed in 400-600 $\mu$ of water at $4^{\circ} \mathrm{C}$. The glands were then crushed in a glass/glass Dounce vessel and the homogenate centrifuged for $30 \mathrm{~min}$ at $10,000 \mathrm{~g}$ at $4^{\circ} \mathrm{C}$. The resulting supernatant was lyophilized and stored at $-80^{\circ} \mathrm{C}$ until required. S. grossa (Brown cupboard spider) venom was extracted from venom sacs dissected from spiders raised in the Neurotoxin Research Group. Spiders were originally obtained by field collection from Gore Hill, Sydney, Australia and were formally identified as S. grossa by $\mathrm{Dr}$ Robert J. Raven of the Queensland Museum (South Brisbane QLD, Australia). Venom was extracted in an identical fashion to that of $L$. hasselti.

A rabbit-derived polyclonal antibody to $\alpha$-LTX raised against the venom of $L$. tredecimguttatus was purchased from Alomone Laboratories. A murine-derived monoclonal antibody to $\alpha$-LTX from the venom of $L$. tredecimguttatus (4C4.1) was produced according to the methods of Pescatori and Grasso [30], and supplied as lyophilized hybridoma supernatant by Dr Alfonso Grasso. This was reconstituted at a concentration of $5 \mu \mathrm{g} / \mathrm{ml}$.

\subsection{Isolated vertebrate bioassays}

Vertebrate toxicity of whole theridiid spider venoms, or venom fractions, were bioassayed using the isolated chick biventer cervicis nerve-muscle (CBCNM) preparation as described previously [37]. All animal experimentation was approved by the Animal Care \& Ethics Committee of the University of Technology, Sydney (RNSH/UTS 0011-036A). In antibody neutralization studies, Latrodectus spp. and $S$. grossa venom samples were pre-treated with $1 \mathrm{ml}$ of reconstituted monoclonal antibody $4 \mathrm{C} 4.1$ solution and allowed to equilibrate with venom for $5 \mathrm{~min}$ at $37^{\circ} \mathrm{C}$ prior to application to the nerve-muscle preparation.

\subsection{Gel electrophoresis and Western blotting}

Western blotting was performed following initial sodium dodecyl sulphate polyacrylamide gel electrophoresis (SDS-PAGE) as described previously [32]. Purified Latrodectus spp. venom fractions were transblotted for subsequent protein sequencing by Edman degradation. The PVDF 
membrane was incubated with $0.025 \%$ Coomassie blue R250 in $40 \%$ methanol for 30 min immediately after transblotting and destained with 50\% methanol. Bands of interest were excised and sent for Edman sequencing (Australian Proteomic Analysis Facility, Macquarie University, Sydney, Australia) on an Applied Biosystems/Perkin Elmer Procise 492 cLC protein sequencer. Secondly, transblotted Latrodectus spp. and S. grossa venom proteins were incubated with polyclonal rabbit-derived anti- $\alpha$-LTX antibody (1:100 dilution) or murine-derived anti- $\alpha-L T X$ monoclonal antibody 4C4.1 [30] as primary antibodies using previously described methods [32]. The blot was then incubated with specific secondary antibodies for $1 \mathrm{~h}$. For the polyclonal anti- $\alpha-$ LTX antibody a 1:10,000 dilution of alkaline phosphate conjugated goat anti-rabbit IgG (SigmaAldrich) was used. For the anti- $\alpha$-LTX monoclonal antibody 4C4.1, a 1:2,000 dilution of alkaline phosphate conjugated goat anti-mouse IgG (Sigma-Aldrich) was employed.

\subsection{Toxin purification and peptide sequencing}

One milligram samples of Latrodectus spp. and S. grossa venoms were initially purified by sizeexclusion fast performance liquid chromatography (FPLC) using a Superdex ${ }^{\circledR} 200$ HR 10/30 FPLC column (1 x $30 \mathrm{~cm}$, Pharmacia-Biotech, Uppsala, Sweden) using a buffer of $20 \mathrm{mM}$ Tris/ $\mathrm{NaCl}, \mathrm{pH}$ 7.6 and a flow rate of 0.5 or $1 \mathrm{ml} / \mathrm{min}$. $\beta$-Galactosidase $(118 \mathrm{kDa})$ was used as an indicator of the retention time as its mass is similar to $\alpha$-LTX. Protein fractions were monitored at 215 and $280 \mathrm{~nm}$ using a Pharmacia LKB variable wavelength monitor model 2141 (Pharmacia-Biotech). Venom fractions were collected manually and stored at $4^{\circ} \mathrm{C}$. Further fractionation of the high molecular mass fractions was performed using anion-exchange FPLC within one day.

Anion-exchange FPLC was performed using a Mono $Q^{\circledR}$ HR 5/5 FPLC column (PharmaciaBiotech). Protein fractions were eluted using $20 \mathrm{mM}$ Tris $/ \mathrm{NaCl}, \mathrm{pH} 7.6$ as the mobile phase, employing a linear $\mathrm{NaCl}$ gradient from 20 to $400-600 \mathrm{mM}$ over $30-35 \mathrm{~min}$ at a flow rate of $1 \mathrm{ml} / \mathrm{min}$. Desalting of anion-exchange FPLC fractions was performed using either a HiTrap ${ }^{\circledR}$ Desalting Column (Pharmacia-Biotech) or using reverse-phase high pressure liquid chromatography (RPHPLC; see below). After desalting, fractions were lyophilized and stored at $-80^{\circ} \mathrm{C}$.

Further separation, prior to Edman sequencing and MS-MS, was performed using an analytical C18 RP-HPLC column (25 cm x $4.6 \mathrm{~mm}, 5 \mu \mathrm{m}$ pore size; Vydac, Hesperia, CA, USA). The column was equilibrated with $95 \%$ buffer $A$ consisting of $0.1 \% \mathrm{v} / \mathrm{v}$ trifluoroacetic acid (TFA) in water and $5 \%$ buffer $B(0.085 \%$ v/v TFA in acetonitrile). Elution of venom components was achieved using a linear gradient of $5-50 \%$ buffer B over 30 min followed by a linear gradient of $50-70 \%$ buffer B over $35 \mathrm{~min}$ at flow rate of $1 \mathrm{ml} / \mathrm{min}$. Purified toxins were collected, lyophilized, and stored at $-80^{\circ} \mathrm{C}$ until required. Toxin quantification was performed using a bicinchoninic acid protein assay kit (Pierce, Rockford, IL, USA) using bovine serum albumin as a standard. Absorbances were read at $560 \mathrm{~nm}$ on a Titertek Multiscan microplate reader (Eflab, Finland). 


\subsection{Acute Insect Toxicity Testing}

For analysis of insecticidal activity, anion-exchange FPLC fractions were dissolved in insect saline with $0.1 \%$ BSA and injected into house crickets (Acheta domesticus) as previously described [38]. Groups of 3-5 crickets received intrathoracic injections at a dose of $1 \mu \mathrm{g} / \mathrm{g}$. A control group of three crickets were also injected with the same volume of $0.1 \%$ BSA in insect saline. Insects were monitored for $96 \mathrm{~h}$ following injection for signs of toxicity, and the percentage knockdown and lethality was determined. Knockdown was defined as the inability to maintain an upright posture, with intermittent or continuous twitches of appendages.

\section{$2.6 \alpha$-LTX fragment sequencing by tandem mass spectrometry}

Purified protein samples were analyzed using electrospray ionization time-of-flight tandem mass spectrometry (ESI-Q-TOF MS). Briefly, samples were purified after fractionation by RP-HPLC using SDS-PAGE gel electrophoresis on 15\% Tris-glycine gels as described above. Gels were stained with $0.025 \%$ Coomassie Blue R-250 in 40\% methanol for 20 to 30 min and destained with $50 \%$ methanol. Protein bands of interest were excised from the gel, dried, and exposed to a $16 \mathrm{~h}$ tryptic digestion at $37^{\circ} \mathrm{C}$ prior to peptide analysis. The resulting peptides were purified using a ZipTip $^{\circledR}$ (Millipore, Bedford, MA, USA) to concentrate and desalt the samples. The samples were then analyzed by tandem mass spectrometry (MS-MS) using a Micromass Q-TOF MS equipped with a nanospray source (Micromass, Manchester, England). Samples were dissolved in $5 \mu \mathrm{l} 50 \%$ acetonitrile and $0.5 \%$ formic acid. Data were manually acquired in the positive mode using borosilicate capillaries with a source temperature of $80^{\circ} \mathrm{C}$. A potential of $850 \mathrm{~V}$ was applied to the nanoflow tip. Data were acquired over the $\mathrm{m} / \mathrm{z}$ range $400-1,800$ to select peptides for MS-MS analysis. After peptides were selected, the MS was switched to MS-MS mode and the data collected over the $\mathrm{m} / \mathrm{z}$ range $50-2,000$ with variable collision energy settings. Peptides were subsequently compared with the known amino acid sequences of $\alpha$-LTX, $\alpha$-latroinsectotoxin, and $\delta$-latroinsectotoxin using $\mathrm{NCBI}$-Blast protein-protein comparison which uses the protein sequences derived from the venom of $L$. tredecimguttatus for sequence homology [8, 10, 39]. Raw data were processed using the MAXENT algorithm included in the MASSLYNX program.

\subsection{Genomic DNA extraction from $L$. hasselti}

Two $L$. hasselti spiders were immobilized by freezing at $-20^{\circ} \mathrm{C}$ for $5 \mathrm{~min}$ and their cephalothoraces dissected. Cell lysis was performed by firstly crushing the cephalothoraces in $500 \mu \mathrm{l}$ of $4 \mathrm{M}$ guanidium isothiocyanate (GITC) (Sigma-Aldrich) in $100 \mathrm{mM}$ Tris (pH 7.0) with $0.1 \% \beta$ mercaptoethanol. Fifty microlitres of $3 \mathrm{M}$ sodium acetate $(\mathrm{pH} 5)$ and $500 \mu \mathrm{l}$ of phenol (saturated solution, $\mathrm{pH}$ 6.6) were then added sequentially to extract the proteins. Subsequent to this, $100 \mu \mathrm{l}$ of chloroform/isoamyl alcohol (49:1) was added to extract the cellular proteins. The solution was centrifuged at $10,000 \mathrm{~g}$ for $10 \mathrm{~min}$ to separate the aqueous and organic layers. The upper layer and genomic DNA (gDNA) interface were carefully pipetted from the protein layer and placed in 
separate tube where DNA precipitation was performed. To the suspension was added two volumes of $100 \%$ ethanol at room temperature. The precipitate was subsequently centrifuged at $10,000 \mathrm{~g}$ for 15 min resulting in the formation of a DNA pellet. The supernatant was decanted from the DNA pellet and the pellet resuspended in $100 \mu \mathrm{ldH} \mathrm{H}_{2} \mathrm{O}$.

gDNA used for the amplification of the segment which comprises bases $3320-3830$ in the nucleotide sequence, was obtained from dissected legs that were ground with a chilled, sterile mortar and pestle in liquid nitrogen. After pulverization, the tissue $(\sim 100 \mathrm{mg})$ was added to $400 \mu \mathrm{l}$ of low salt buffer as previously described [40]. Before amplification by the Polymerase Chain Reaction (PCR), quality and quantity of all extracted DNA was assessed by measuring the OD260/280 and by running a $1.1 \%$ agarose gel, stained with SyberSafe ${ }^{\circledR}$.

\subsection{PCR and Sequencing}

Non-degenerate paired forward and reverse DNA primers were designed from the previously determined DNA sequence of $L$. tredecimguttatus $\alpha$-LTX [8] and peptide sequences derived from MS-MS sequencing. Primer pairs were selected at approximately 400-600 bp distances and Primerfinder (http://eatworms.swmed.edu/ tim/primerfinder/) was utilized to reduce the likelihood of primer dimers or hairpins. Primers were supplied by Sigma GenoSys, Australia.

Red-Taq DNA Polymerase (Sigma) was used to amplify all segments of the $\alpha$-LTX from L. hasselti by PCR, except for the segment at position 3320-3830 bp which was amplified using Platinum ${ }^{\circledR}$ Taq (Invitrogen). Each $50 \mu \mathrm{l}$ reaction contained 250-300 ng of gDNA template, 1X Sigma PCR buffer (or 1X PCR reaction buffer and $1 \mathrm{mM} \mathrm{MgCl}$ ), $0.2 \mu \mathrm{M}$ dNTPs, $0.6 \mu \mathrm{M}$ of each forward and reverse primer, $1 \mathrm{U}$ Taq Red-Taq polymerase (or $1 \mathrm{U}$ Platinum Taq polymerase). Cycling conditions were: $1 \mathrm{~min}$ at $94^{\circ} \mathrm{C}$ followed by 35 cycles of $94^{\circ} \mathrm{C}$ for $30 \mathrm{~s}, 50^{\circ} \mathrm{C}$ for $30 \mathrm{~s}, 72^{\circ} \mathrm{C}$ for 1 min and a final cycle of $72^{\circ} \mathrm{C}$ for $10 \mathrm{~min}$.

The PCR products were purified using the QIAGEN QIAquick PCR purification kit and sent directly for sequencing whereas clean PCR products from the segment 3320-3830bp were cloned into the $\mathrm{pCR} \|^{\circledR}$ vector using the TA dual Cloning Kit (Invitrogen). The resulting clones were grown overnight in LB media with ampicillin. Plasmids were extracted using the QIAprep Spin Miniprep kit. Sequencing was performed at the Sydney University Prince Alfred Macromolecular Analysis Centre (SUPAMAC) where samples were prepared and mixed with 70 to 100 pmol of respective forward or reverse primers. In later experiments sequencing was performed at the Australian Genome Research Facility (AGRF - Brisbane node) in a reaction containing $3 \mu \mathrm{l}$ of Plasmid DNA with M13 primers. Sequences were aligned and analyzed using MACVECTOR (v11.0.4) or GENEIOUS PRO software (v4.8.3)

Given the similar, but non-identical, sequences of the $\alpha$-LTX peptides determined in this work 
when compared to $\alpha$-LTXs from $L$. tredecimguttatus, it is proposed that a new nomenclature system be employed to describe the $\alpha$-LTX homologs from other Latrodectus venoms. Accordingly, we used a nomenclature based on that recently described for venom peptides [41] and which has already been adopted by ArachnoServer [42, 43] and UniProtKB [44]. Hence, $\alpha$-latrotoxin from $L$. tredecimguttatus is renamed $\alpha$-latrotoxin-Lt1a $(\alpha$-LTX-Lt1a) and $\alpha$-latrotoxins from the venom of $L$. hasselti, $L$. hesperus and $L$. mactans are named $\alpha-L T X-L h 1 a, \alpha-L T X-L h e 1 a$ and $\alpha-L T X-L m 1 a$, respectively, in order to clearly distinguish them from $\alpha-L T X-L t 1 a$.

\section{RESULTS}

\subsection{In vitro monoclonal antibody neutralization studies}

The venoms from L. tredecimguttatus, L. lugubris, L. hesperus and L. hasselti (Fig. 1, top panels) all produced a characteristic rapid muscle contracture and loss of twitch tension when added to the isolated and indirectly-stimulated chick biventer cervicis nerve-muscle (CBCNM) preparation. This is consistent with the known ability of $\alpha$-LTX to increase neurotransmitter release in this preparation [32, 45]. The murine-derived monoclonal antibody 4C4.1, raised against $\alpha$-LTX-Lt1a from $L$. tredecimguttatus, was then assayed for its ability to inhibit the in vitro effects of these Latrodectus venoms. The responses of $L$. tredecimguttatus venom on twitch tension and contracture were inhibited by $4 \mathrm{C} 4.1$ in a dose-dependent fashion. Following a 5 min incubation with $1 \mathrm{ml}$ of $4 \mathrm{C} 4.1(5 \mu \mathrm{g} / \mathrm{ml})$ at $37^{\circ} \mathrm{C}$, L. tredecimguttatus venom failed to alter twitch tension (Fig. 1Ab). Incubation with reduced amounts of $4 \mathrm{C} 4.1(<2.5 \mu \mathrm{g} / \mathrm{ml})$ resulted in the production of a moderate contracture and slow, but eventual, loss of twitch tension (data not shown). In contrast, incubation with $1 \mathrm{~mL}$ of $4 \mathrm{C} 4.1$ failed to prevent the typical, rapid contracture associated with diminishing twitch tension associated with L. lugubris, L. hesperus, and $L$. hasselti venoms (Fig. 1Bb, $\mathrm{Cb}$ and $\mathrm{Db}$ ). These observations suggest that either the concentration of antibody was insufficient to completely block the neurotoxic effects of these latter venoms or more likely that the epitope that binds 4C4.1 may not be present in the $\alpha$-LTX-like molecules found in other Latrodectus venoms.

\subsection{Venom electrophoresis and Western blotting}

SDS-PAGE revealed comparable high molecular mass proteins for all Latrodectus spp. venoms with a protein band around $130 \mathrm{kDa}$, consistent with the presence of $\alpha$-LTX (Fig. 2A). Subsequent Western blotting with polyclonal anti- $\alpha$-LTX-Lt1a antibody revealed that this 130-kDa band present in all venoms has antigenic similarity to $\alpha$-LTX-Lt1a from $L$. tredecimguttatus (Fig. 2B). In support, Western blotting with the monoclonal antibody 4C4.1 showed binding with $\alpha$-LTX-Lt1a derived from $L$. tredecimguttatus venom as well as the $130-\mathrm{kDa}$ band found in $L$. tredecimguttatus (Fig. 2C). Interestingly, however, no binding was observed with any protein bands found in L. hasselti, L. mactans, L. hesperus, or with the $\alpha$-LTX-like protein previously described in 
S. grossa venom [46], (Fig. 2C) or with L. lugubris (data not shown). These results are consistent with the inability of $4 \mathrm{C} 4.1$ to neutralize the neurotoxicity of $L$. hasselti, $L$. hesperus, and $L$. lugubris venoms. This indicates that the epitope responsible for binding $4 \mathrm{C} 4.1$ is either not present, or is modified, in the $\alpha$-LTX molecules found in other Latrodectus venoms and suggests that the protein structure of these toxins may vary from the known structure of $\alpha$-LTX-Lt1a [47].

\subsection{Size-exclusion FPLC}

Bioassay-guided fractionation of Latrodectus and S. grossa venoms was undertaken to provide proteins for $\mathrm{N}$-terminal sequencing. Initial separation of venoms was undertaken using size exclusion FPLC employing isocratic elution with $20 \mathrm{mM}$ Tris/ $\mathrm{NaCl}(\mathrm{pH}$ 7.6). These conditions are similar to that used previously for the separation of $\alpha$-LTX-Lt1a from $L$. tredecimguttatus venom [48]. The chromatograms resulting from fractionation of the venoms of $L$. tredecimguttatus, L. lugubris, L. hasselti, and S. grossa all contained a high molecular mass fraction ( 200 kDa) eluting at 8-12 min (Fig. 3A-D), a fraction containing intermediate masses (120-130 kDa) eluting at 12-18 $\mathrm{min}$, and a number of lower molecular mass proteins eluting at longer retention times (Fig. $3 \mathrm{H}$ ). In contrast, the venoms from L. mactans and L. hesperus were almost completely devoid of the high molecular mass fraction (Fig. 3E-F) and had reduced expression of lower molecular mass proteins (Fig. $3 \mathrm{H})$; these venoms were instead dominated by the intermediate-mass fraction (shaded black in each of the chromatograms in Fig. 3). The early-eluting high-mass fraction may represent contaminant proteins extracted from the venom gland itself, although this fraction is present in the venom from $L$. lugubris milked by electrostimulation. The intermediate-mass fraction had a similar retention time to $\beta$-galactosidase (118 kDa; Fig. $3 G$ ), suggesting that it likely contains $\alpha$-LTX-like molecules. Consistent with this hypothesis, these fractions exhibited an increased muscle contracture and decreased twitch tension similar to crude venom extracts when bioassayed using the CBCNM preparation (data not shown). Interestingly, the 120-130 kDa size-exclusion fraction from $S$. grossa venom exhibited much less activity in the nerve-muscle bioassay with minimal increases in muscle contracture seen in the absence of any changes in twitch tension. This is consistent with the observations made earlier in comparisons of the bioactivity of crude venom from this species with that of Latrodectus spp. venoms [46].

Initial attempts at using C18 RP-HPLC fractionation employing 0.1\% TFA in acetonitrile resulted in loss of activity of resultant venom fractions bioassayed using the CBCNM preparation. This was most likely due to the acidic/denaturing environment resulting in neutralization of toxin activity. Therefore all 120-130 kDa venom fractions were subsequently purified by anion exchange FPLC.

\subsection{Anion Exchange FPLC}

The intermediate-mass FPLC fractions exhibiting neurotoxic actions in the CBCNM bioassay were further separated by anion-exchange FPLC using a $\mathrm{NaCl}$ gradient. All Latrodectus spp. and 
S. grossa venoms exhibited a large number of fractions that sequentially eluted with increasing $\mathrm{NaCl}$ concentration (Fig. 4A-F). Latrodectus venoms all contained a vertebrate-toxic fraction as determined by the CBCNM bioassay that resulted in a typical increase in resting muscle tension and loss of twitch tension (Fig. 4G-J) comparable to that observed with crude venom extract (Fig. 1Aa-Da). This was invariably found in the final peak eluting at a $\mathrm{NaCl}$ concentration of 360$370 \mathrm{mM} \mathrm{NaCl}$ for L. tredecimguttatus $(f 6)$ and L. Iugubris $(f 6)$, consistent with that previously seen for $L$. tredecimguttatus [11], and 440-460 mM for L. mactans $(f 6), L$. hesperus $(f 6)$ and $L$. hasselti (f7) (black peaks in Fig. 4A-E). SDS-PAGE of these anion-exchange fractions revealed that they contained at least one high-molecular mass protein band (120-130 kDa) with some also containing $70-\mathrm{kDa}$ contaminants (Fig. 5A-C). An attenuated response in the nerve-muscle preparation was also seen with the fraction immediately preceding these final fractions suggesting that there was some degree of cross-contamination of these venom fractions with the $\alpha$-LTX-like protein found in the final fraction of each venom (data not shown). Furthermore, acute insect toxicity was observed in the L. hasselti $(f 6), L$. mactans $(f 5), L$. hesperus $(f 5)$, and L. lugubris $(f 5)$ anion-exchange fractions (white peaks in Fig. 4B-E) preceding the vertebrate-toxic fraction (black peaks in Fig. 4AE). In the insect bioassays, 30-100\% knockdown was observed at $24 \mathrm{~h}$ postinjection, with $100 \%$ lethality at $96 \mathrm{~h}$. The insect toxicity of these fractions suggests that the high-molecular weight bands seen on SDS-PAGE may contain latroinsectotoxin homologs.

Attempts to isolate the vertebrate-active fraction of $S$. grossa venom using a similar method to that employed for the Latrodectus venoms were unsuccessful. Although the high molecular weight size exclusion fractions of the venom showed limited activity, the various anion-exchange fractions purified from the size-exclusion fractions failed to elicit a response in the CBCNM preparation. This suggests that the $S$. grossa anion-exchange venom fractions may have become inactivated during the fractionation process. Hence, further attempts at isolation and characterization of $S$. grossa toxins was not attempted.

\subsection{N-terminal sequencing of $\alpha$-LTX-associated low molecular weight proteins}

$\mathrm{N}$-terminal sequencing via Edman degradation was attempted using the vertebrate-active final peak of all the Latrodectus spp. venoms fractionated by anion-exchange FPLC. Initial attempts to sequence $\alpha$-LTX from the vertebrate-active anion-exchange FPLC peak of $L$. tredecimguttatus $(f 6)$ venom proved unsuccessful, with only a partial sequence of a co-eluting low-molecular weight protein (LMWP, also named latrodectin) associated with $\alpha$-LTX [17-19, 21, 47] being identified (see Supplementary Fig. S1). Similarly, a partial N-terminal sequence of LMWP was identified from the corresponding vertebrate-active fraction $(f 6)$ of L. Iugubris venom (Supplementary Fig. S1). This showed $100 \%$ identity with the reported sequence of LMWP from L. tredecimguttatus, providing further support for the contention that $L$. lugubris is not a separate species from $L$. tredecimguttatus [36]. When sequencing was attempted using toxin resolved on SDS-PAGE and then transblotted to 
PVDF membrane, LMWP was not detected. This suggests that SDS-PAGE followed by transblotting results in separation of the high ( $\alpha-L T X)$ and low molecular mass (LMWP) proteins. We were unable to determine any similar sequences for LMWP from any other Latrodectus venoms either when on blot or direct sequencing of toxin were attempted.

Attempts at $\mathrm{N}$-terminal sequencing of $\alpha$-LTX from the remaining vertebrate-active fractions of L. hasselti, L. mactans, or L. hesperus venom also failed when attempted in-solution or when transblotted onto PVDF membrane, suggesting that these toxins may be N-terminally blocked [39]. Given that the N-terminal residue of $\alpha$-LTX-Lt1a is glutamate, $N$-terminal block may result from the conversion to pyroglutamate [49], which is resistant to $\mathrm{N}$-terminal sequencing by Edman degradation [50]. As a result, the vertebrate-active peaks of $L$. hasselti, L. mactans, L. lugubris and L. hesperus venoms were subjected to SDS-PAGE and on-blot pyroglutamate aminopeptidase digestion in an attempt to cleave any N-terminal pyroglutamate. This method was unsuccessful suggesting that either the toxin may not have been present in sufficient quantities to be detected by this sequencing method, or that alternative post-translational modifications that interfere with Edman sequencing may be present [50].

\subsection{MS-MS protein sequencing of latrotoxins}

To a derive a partial amino acid sequence of $\alpha$-LTX homologs from Latrodectus venoms, MS-MS protein sequencing of tryptic-digest fragments of the vertebrate-active fractions was carried out. Initially, MS-MS sequencing was used to analyze tryptic-digest peptides from L. hasselti and L. hesperus vertebrate-active anion-exchange fractions $f 7$ and $f 6$, respectively. Interestingly, the peptides sequenced were highly homologous with $\alpha$-LIT and $\delta$-LIT $[9,10]$ and not $\alpha-L T X$ (Supplementary Fig. S2). Results are summarized in Table 1. This most likely arose as a result of significant cross-contamination with the immediately preceding latroinsectotoxin anion-exchange FPLC fractions ( $f 6$ and $f 5$, respectively). However, it would not be possible to further separate these contaminants by size-exclusion FPLC or gel electrophoresis due to the similarity in the molecular masses of the toxins. Hence, further purification of $\alpha$-LTX from the vertebrate-active anion-exchange FPLC fractions from $L$. hasselti $(f 7)$, L. hesperus $(f 6)$, and $L$. mactans $(f 6)$ venoms was achieved using C18 RP-HPLC (Fig. 5D-F). Reverse-phase HPLC was not used earlier in the purification process as we had previously found that the mobile phase inactivated crude venom and venom fractions making it impossible to use the CBCNM bioassay to determine which fraction was vertebrate-active.

C18 RP-HPLC fractionation of the vertebrate-active anion-exchange fraction from L. mactans and L. hesperus revealed nine and seven sub-fractions, respectively (Fig. 5D-E). For L. hesperus, three fractions contained proteins with a molecular mass of $120 \mathrm{kDa}$ when separated on SDS-PAGE. Two of these were excised and subjected to a $16 \mathrm{~h}$ tryptic digestion. MS-MS analysis of two of 
these peptide fragments revealed sequences homologous to $\alpha$-LTX in $f 6.6$, and $\delta$-latroinsectotoxin in $f 6.4$ (not analyzed further in view of previous amino acid sequence analysis). Although only two peptide sequences (22 amino acids in total) were determined, they exhibited $100 \%$ identity with $\alpha$-LTX-Lt1a (Fig. 6, Table 1).

For L. mactans, C18 RP-HPLC fractionation and subsequent SDS-PAGE analysis revealed two fractions ( $f 6.8$ and $f 6.9$ ) containing a molecular mass of 120-130 kDa. Following tryptic digestion, both fractions contained peptide fragments with high homology with $\alpha$-LTX-Lt1a. Fraction 6.8 revealed 13 peptides containing 150 amino acids with $94.6 \%$ identity and $98 \%$ similarity with $\alpha$ LTX-Lt1a. Fraction 6.9 returned 10 peptides containing a total of 119 amino acids with $91.6 \%$ identity and $95.7 \%$ similarity with $\alpha$-LTX-Lt1a (Fig. 6, Table 1). A number of peptides sequenced from these two fractions were identical. It is interesting that two $\alpha-L T X$ homologs were purified from L. mactans anion exchange vertebrate-active fraction by RP-HPLC. This suggests that there might be two distinct $\alpha$-LTX isoforms in L. mactans venom with similar but not identical primary structure.

Finally, L. hasselti $f 7$ yielded nine RP-HPLC fractions (Fig. 5F). Two fractions ( $f 7.7$ and $f 7.9$ ) yielded 120-130 kDa bands when separated on SDS-PAGE. These were excised, subjected to tryptic digestion, and analyzed using MS-MS. No peptides could be identified in $f 7.7$. However, f7.9 yielded four peptide sequences (total of 54 amino acids) that yielded a $100 \%$ sequence match with $\alpha$-LTX-Lt1a [8] (Fig. 6, Table 1).

\subsection{Determination of the DNA sequence of Latrodectus hasselti $\alpha$-LTX gene}

It was difficult to reconcile the $100 \%$ identity of the $L$. hasselti $f 7.9$ peptide fragments and $\alpha$-LTXLt1a with the observed lack of neutralization using the anti- $\alpha-L T X-L t 1$ a monoclonal antibody 4C4.1. We therefore decided to construct a $L$. hasselti genomic DNA library in order to be able to determine the complete sequence of the gene encoding the $\alpha$-LTX-like protein. The choice of Latrodectus species was based on the ready availability of $L$. hasselti and the fact that it is a medically significant spider, associated with significant morbidity; this spider is estimated to be responsible for at least 5,000 envenomations per year in Australia [2].

Determination of the DNA sequence of $\alpha$-LTX-Lh1a from the genomic DNA of this spider was performed in a stepwise fashion along the $\alpha$-latrotoxin gene. This process resulted in the definitive determination of 3845 bases, covering the complete mature toxin sequence, including a three base insertion (bases +10-12, Fig. 7). Comparison with $\alpha$-LTX-Lt1a revealed that the DNA sequence also covered $35 \%$ of the signal peptide and $81 \%$ of the C-terminal propeptide region of $\alpha$-LTX-Lt1a [11]. There was $95.1 \%$ sequence identity between the DNA sequence encoding $\alpha$-LTX-Lh1a with that encoding the mature $\alpha$-LTX-Lt1a toxin (Fig. 7). 
Translation of the genomic DNA sequence for $\alpha$-LTX-Lt1a yielded a mature toxin comprising 1180 amino acid residues with a predicted molecular mass of 132,514 Da and a p/ of 5.72. 1097 (93\%) of these residues are identical to those in the mature $\alpha$-LTX-Lt1a toxin, which increases to $93.2 \%$ if three residues identical to a variant of $\alpha$-LTX-Lt1a (AAC78471 in EMBL-Bank CDS) are included (V41, V143 and D1097). Of the 83 amino acid substitutions, 34 are conservative and 49 nonconservative, resulting in an overall $93.8 \%$ similarity with $\alpha$-LTX-Lt1a (Fig. 8). Importantly the deduced polypeptide contained all four partial amino acid sequences previously sequenced by tandem MS of $f 7.9$. Furthermore, the mature $\alpha$-LTX-Lh1a also contains two hydrophobic segments in its N-terminal region, HS1 (residues 35-60) and HS2 (219-238) homologous to similar segments found in $\alpha-$ LTX-Lt1a (Fig. 8) and $\delta$-LIT [10].

Analysis of the deduced amino acid sequence revealed the presence of 22 imperfect repeats (R1R22) at positions 439-1180 (Fig. 8). These comprise the ankyrin-like repeat motif previously described in $\alpha$-LTX-Lt1a [8] and other latrotoxins $[9,10,16]$ and divide the mature toxin into two domains: the N-terminal domain II (50,026 Da, 38\%) and the ankyrin-rich domain III (82,506 Da, $62 \%$ ). Ankryin repeats are a sequence motif found in proteins with various functions including transcription initiation, cell cycle regulation, cytoskeletal integrity, ion transport, and cell-cell signaling [51]. These repeats have a consensus sequence as shown in Supplementary Fig. S2A $[52,53]$. The 33-residue ankyrin repeats in $\alpha$-LTX-Lh1a have a highly conserved length, except for $\mathrm{R} 1, \mathrm{R} 16$ and $\mathrm{R} 17$ (32 residues); similarity to the consensus sequence ranges from 9\% (R16) to $61 \%$ (R3 and R13). Analogous to $\alpha$-LTX-Lt1a, repeats R1, R16 and R17 are atypical, imperfect repeats (Supplementary Fig. S2B). Finally, the putative binding site for $4 \mathrm{C} 4.1$ was derived from the DNA sequence for $\alpha$-LTX-Lh1a (nucleotide bases 3156-3177). Importantly, $\alpha$-LTX-Lh1a contains non-conservative $\mathrm{F} 1009 \mathrm{D}$ and $\mathrm{S} 1011 \mathrm{~N}$ substitutions in the binding epitope when compared to $\alpha$ LTX-Lt1a.

\section{DISCUSSION}

Comparative Western blot analysis of Latrodectus spp. and S. grossa venoms using a polyvalent anti- $\alpha$-LTX-Lt1a antibody revealed that all venoms contained proteins that are antigenically similar to those found in L. tredecimguttatus, in particular a $130 \mathrm{kDa}$ band consistent with $\alpha-L T X$. The pattern of expression is similar to that seen in Western blots employing polyvalent red-back spider antivenom, an equine-derived antibody composed of purified IgG-F(ab') $)_{2}$ fragments produced from the venom of $L$. hasselti $[32,46]$. These findings suggested the presence of an $\alpha$-LTX-Lt1a-like protein in all Latrodectus spp. and S. grossa venoms. The subsequent identification of $\alpha$-LTX and LIT sequences from Latrodectus venoms, other than L. tredecimguttatus, provides the first confirmation that $\alpha$-LTXs are widely expressed in theridiid spiders. 
Using non-degenerate primers based on the DNA sequence of $\alpha$-LTX-Lt1a and MS-MS peptide sequences from $L$. hasselti RP-HPLC fractions, we were also able to determine the complete sequence of the novel $\alpha$-LTX-Lh1a from the venom of the red-back spider $L$. hasselti. The primary structure of $\alpha$-LTX-Lh1a has the same domain structure as $\alpha$-LTX-Lt1a. $\alpha$-LTX-Lh1a contains two furin-like endopeptidase cleavage sites coded for by a cluster of 4 basic residues: 'RMRR' at the Nterminus ( -4 to -1$)$ and 'KFRR' at the C-terminus (1177-1180) followed by an acidic amino acid (glutamate). These conform to the $(K / R) \Phi_{1-3}(K / R)_{0-1} R$, consensus sequence for proteolytic cleavage of $\alpha$-LTX-Lt1a and LITs where $K$ and R are lysine and arginine, respectively, and $\Phi$ is any hydrophobic/aromatic residue [54]. These furin sites are believed to be important for removal of the signal peptide and C-terminal propeptide in $\alpha$-LTX-Lt1a [11]. Therefore $\alpha$-LTX-Lh1a appears to be produced as a preprotoxin in a similar fashion to $\alpha$-LTX-Lt1a. Unfortunately most of the nucleotide sequence upstream of the $\mathrm{N}$-terminus of the mature protein could not be determined and therefore we cannot draw any conclusions about the size of the signal peptide and its homology to that of $\alpha$-LTX-Lt1a. We sequenced 160 bases in the domain IV propeptide region but unfortunately we were unable to sequence to the stop codon 201 residues downstream of the furin cleavage site in $\alpha-L T X-L t 1 a$. However, within domain II and III, the cysteine residues important for structural stability and activity of latrotoxins $[20,55]$ as well as the two hydrophobic segments in domain II, were all conserved. In particular, the second hydrophobic segment (HS2) is highly conserved between $\alpha$-LTX-Lh1a and all latro(insect/crusta)toxins (see [10]) and may, therefore, be involved in an important aspect of function in all latrotoxins.

Despite the fact that all the venoms tested appeared to be antigenically similar when probed with polyclonal anti- $\alpha$-LTX antibodies, the anti- $\alpha$-LTX-Lt1a monoclonal antibody $4 C 4.1$ failed to recognize venom from $L$. hasselti, $L$. hesperus, $L$ mactans, and $S$. grossa. This was noted in both Western blots and in the inability of $4 \mathrm{C} 4.1$ to prevent the rapid muscle contracture and loss of twitch tension produced by these latter venoms in the CBCNM preparation. Thus, even though all Latrodectus and S. grossa venoms appear to contain $\alpha$-LTX-like proteins, the epitope required for binding of the 4C4.1 antibody appears to be present only in the toxin from L. tredecimguttatus, or is at least significantly modified in the $\alpha$-LTX-like toxins from other Latrodectus/Steatoda venoms. The putative binding site for $4 \mathrm{C} 4.1$ was determined directly by tandem MS from L. mactans f7.9 ( $\alpha$-LTX-Lm1a) and derived from the DNA sequence for $\alpha$-LTX-Lh1a. Both $\alpha$-LTX-Lm1a and $\alpha$-LTXLh1a contain a non-conservative F1028D substitution when compared to $\alpha$-LTX-Lt1a. In addition, $\alpha$-LTX-Lh1a also contains a non-conservative $S 1030 \mathrm{~N}$ substitution. These substitutions explain the lack of binding of $4 \mathrm{C} 4.1$ to L. hasselti and L. mactans protein bands in Western blots, and also the lack of neutralizing activity of the antibody in the chick nerve-muscle preparation. L. hesperus $\alpha$ LTX-Lhe1a is also likely to contain similar substitutions that underlie the ineffectiveness of 4 C4.1 in Western blots and neutralization assays. 
The actions of $\alpha$-LTX-Lh1a to cause rapid spontaneous neurotransmitter release, leading to an inhibition of neurotransmission at the chick neuromuscular junction, are indistinguishable from that of $\alpha$-LTX-Lt1a (Fig. 4G-I). This results from the known action of LTXs to cause massive vesicle exocytosis [4-6]. This action is consistent with the clinical reports of latrodectism and animal studies using $L$. hasselti venom, including reports of muscle fasciculation, tremor, muscle paralysis, tachycardia, priapism, hypertension, salivation and diaphoresis resulting from excessive neurotransmitter release from motor and autonomic nerve endings [2, 32]. Since $\alpha$-LTX-Lt1a shares a very high degree of sequence homology with $\alpha$-LTX-Lh1a, and probably $\alpha$-LTX-Lm1a and $\alpha$-LTX-Lhe1a, this indicates that the subtle differences in structure between these $\alpha$-LTX homologs do not significantly alter the intense neurotransmitter release seen in vitro with all Latrodectus venoms ([32] and Fig. 4G-I). These findings therefore provide strong support for the widely held assumption that the clinical symptoms of latrodectism are due to the presence of $\alpha$-LTX homologs in all theridiid venoms.

The in vitro, in vivo and clinical observations of the effectiveness of red-back spider antivenom in the treatment of latrodectism from a wide range of Latrodectus species [32-35] suggests that it may therefore be possible to use a single antivenom worldwide for the treatment of widow-spider envenomation. This is likely to impact less on regions that already have their own antivenom to treat envenomation by local Latrodectus species [56], but could potentially benefit many regions of the world where specific antivenoms to local widow spiders are not available. Given the success of red-back spider antivenom in neutralizing the activity of a variety of Latrodectus venoms [32], together with the efficacy of the monoclonal antibody 4C4.1 [31] and Fab antibody fragment FM1 [57] (also see [58]) in neutralizing the activity of whole L. tredecimguttatus venom or $\alpha$-LTX-Lt1a, there has been some interest in the development of monoclonal antibody therapy for the treatment of widow spider antivenom. The advantages of this approach include the virus- and sterile-free methods of production and the high purity of recombinant proteins lacking contaminant animal proteins derived from animal sera. Also, when compared to the large molecular mass IgG-based polyvalent antivenoms, Fab fragment antibodies are likely to diffuse more rapidly due to their smaller molecular mass, and distribute more extensively due to a larger apparent volume of body distribution. These may result in more rapid inactivation of circulating toxin and an earlier recovery from persistent symptoms of latrodectism.

However, as evidenced by neutralization studies in the CBCNM preparation, the monoclonal antibody $4 \mathrm{C} 4.1$ is unlikely to have any utility as an antidote in the treatment of latrodectism from any species other than $L$. tredecimguttatus. Monoclonal antibodies may not bind or neutralize $\alpha$ LTXs from other species, reflective of the highly homologous but not identical primary structure of $\alpha$-LTXs across the family Theridiidae. Furthermore, in view of the mechanism of action of the $4 C 4.1$ antibody, it is unlikely that it would be an effective treatment for established envenomation. 
Pescatori et al. have shown that while 4C4.1 exerts its effect through prevention of tetramerization of $\alpha$-LTX-Lt1a prior to its insertion into cell membranes, once $\alpha$-LTX-Lt1a tetramer ion channels have been formed the antibody is incapable of reversing $\alpha$-LTX-Lt1a pore formation [30]. Hence, polyclonal anti- $\alpha-L T X$ antibodies targeting multiple epitopes on the toxin are likely to be more effective at preventing and reversing the toxicity of widow-spider venoms. Furthermore, in view of the greater expense in production of specific antitoxins, production of polyclonal antivenoms reared in animals exposed to crude venoms remains the least expensive option for the production of clinically useful antivenoms.

\section{ACKNOWLEDGEMENTS}

The authors would like to thank M. Pescatori and A. Grasso, Istituto di Biologia Cellulare, CNR, Rome, Italy for providing the monoclonal antibody 4C4.1. This work was supported by a Rotary Health Research Grant to GMN, AG, and KWB. SSP was supported by a University of Queensland Endeavour International Postgraduate Research Scholarship.

\section{REFERENCES}

[1] Maretic Z. Latrodectism: variations in clinical manifestations provoked by Latrodectus species of spiders. Toxicon 1983;21:457-66.

[2] Isbister G, White J. Clinical consequences of spider bites: recent advances in our understanding. Toxicon 2004;43:477-92.

[3] Grasso A. Preparation and properties of a neurotoxin purified from the venom of black widow spider (Latrodectus mactans tredecimguttatus). Biochim Biophys Acta 1976;439:406-12.

[4] Krasnoperov V, Bittner MA, Holz RW, Chepurny O, Petrenko AG. Structural requirements for $\alpha$-latrotoxin binding and $\alpha$-latrotoxin-stimulated secretion. A study with calcium-independent receptor of $\alpha$-latrotoxin (CIRL) deletion mutants. J Biol Chem 1999;274:3590-6.

[5] Ceccarelli B, Grohovaz F, Hurlbut WP. Freeze-fracture studies of frog neuromuscular junctions during intense release of neurotransmitter. I. Effects of black widow spider venom and $\mathrm{Ca}^{2+}$-free solutions on the structure of the active zone. J Cell Biol 1979;81:163-77.

[6] Longenecker HJ, Hurlbut W, Mauro A, Clark A. Effects of black widow spider venom on the frog neuromuscular junction. Effects on end-plate potential, miniature end-plate potential and nerve terminal spike. Nature 1970;225:701-3. 
[7] Orlova EV, Rahman MA, Gowen B, Volynski KE, Ashton AC, Manser C, et al. Structure of a-latrotoxin oligomers reveals that divalent cation- dependent tetramers form membrane pores. Nature Struct Biol 2000;7:48-53.

[8] Kiyatkin NI, Dulubova IE, Chekhovskaya IA, Grishin EV. Cloning and structure of cDNA encoding $\alpha$-latrotoxin from black widow spider venom. FEBS Lett 1990;270:127-31.

[9] Kiyatkin NI, Dulubova IE, Grishin EV. Cloning and structural analysis of $\alpha$-latroinsectotoxin cDNA. Abundance of ankyrin-like repeats. Eur J Biochem 1993;213:121-7.

[10] Dulubova IE, Krasnoperov VG, Khvotchev MV, Pluzhnikov KA, Volkova TM, Grishin EV, et al. Cloning and structure of $\delta$-latroinsectotoxin, a novel insect-specific member of the latrotoxin family: functional expression requires C-terminal truncation. J Biol Chem 1996;271:7535-43.

[11] Volynski KE, Nosyreva ED, Ushkaryov YA, Grishin E. Functional expression of a-latrotoxin in baculovirus system. FEBS Lett 1999;442:25-8.

[12] Grishin EV. Black widow spider toxins: the present and the future. Toxicon 1998;36:1693701.

[13] Krasnoperov VG, Shamotienko OG, Grishin EV. Isolation and properties of insect and crustacean-specific neurotoxins from the venom of the black widow spider (Latrodectus mactans tredesimguttatus). J Nat Toxins 1992;1:17-23.

[14] Krasnoperov VG, Shamotienko OG, Grishin EV. A crustacean-specific neurotoxin from the venom of the black widow spider Latrodectus mactans tredecimguttatus. Bioorganicheskaia Khimiia 1990;16:1567-9.

[15] Volynskii KE, Volkova TM, Galkina TG, Krasnoperov VG, Pluzhnikov KA, Khvoshchev MV, et al. [Molecular cloning and primary structure of cDNA fragment for a-latrocrustatoxin from black widow spider venom]. Bioorganicheskaia Khimiia 1999;25:25-30.

[16] Danilevich VN, Luk'ianov SA, Grishin EV. [Cloning and structure of gene encoded $\alpha-$ latrocrustoxin from the Black widow spider venom]. Bioorganicheskaia Khimiia 1999;25:537-47.

[17] Gasparini S, Kiyatkin N, Drevet P, Boulain JC, Tacnet F, Ripoche P, et al. The low molecular weight protein which co-purifies with a-latrotoxin is structurally related to crustacean hyperglycemic hormones. J Biol Chem 1994;269:19803-9.

[18] Volkova TM, Pluzhnikov KA, Woll PG, Grishin EV. Low molecular weight components from black widow spider venom. Toxicon 1995;33:483-9. 
[19] Pescatori M, Bradbury A, Bouet F, Gargano N, Mastrogiacomo A, Grasso A. The cloning of a cDNA encoding a protein (latrodectin) which co-purifies with the a-latrotoxin from the black widow spider Latrodectus tredecimguttatus (Theridiidae). Eur J Biochem 1995;230:322-8.

[20] Kiyatkin NI, Kulikovskaya IM, Grishin EV, Beadle DJ, King LA. Functional characterization of black widow spider neurotoxins synthesised in insect cells. Eur J Biochemistry 1995;230:854-9.

[21] Grishin EV, Himmelreich NH, Pluzhnikov KA, Pozdnyakova NG, Storchak LG, Volkova TM, et al. Modulation of functional activities of the neurotoxin from black widow spider venom. FEBS Lett 1993;336:205-7.

[22] Ushkaryov YA, Petrenko AG, Geppert M, Sudhof TC. Neurexins: synaptic cell surface proteins related to the $\alpha$-latrotoxin receptor and laminin. Science 1992;257:50-6.

[23] Petrenko AG, Kovalenko VA, Shamotienko OG, Surkova IN, Tarasyuk TA, Ushkaryov Yu A, et al. Isolation and properties of the $\alpha$-latrotoxin receptor. EMBO $\mathrm{J}$ 1990;9:2023-7.

[24] Davletov BA, Shamotienko OG, Lelianova VG, Grishin EV, Ushkaryov YA. Isolation and biochemical characterization of a $\mathrm{Ca}^{2+}$-independent $\alpha$-latrotoxin-binding protein. $\mathrm{J}$ Biol Chem 1996;271:23239-45.

[25] Krasnoperov VG, Beavis R, Chepurny OG, Little AR, Plotnikov AN, Petrenko AG. The calcium-independent receptor of $\alpha$-latrotoxin is not a neurexin. Biochem Biophys Res Comm 1996;227:868-75.

[26] Sugita S, Ichtchenko K, Khvotchev M, Sudhof TC. $\alpha$-Latrotoxin receptor CIRL/latrophilin 1 (CL1) defines an unusual family of ubiquitous G-protein-linked receptors. G-protein coupling not required for triggering exocytosis. J Biol Chem 1998;273:32715-24.

[27] Ichtchenko K, Bittner MA, Krasnoperov V, Little AR, Chepurny O, Holz RW, et al. A novel ubiquitously expressed $\alpha$-latrotoxin receptor is a member of the CIRL family of G-protein-coupled receptors. J Biol Chem 1999;274:5491-8.

[28] Krasnoperov V, Bittner MA, Mo W, Buryanovsky L, Neubert TA, Holz RW, et al. Proteintyrosine phosphatase- $\sigma$ is a novel member of the functional family of $\alpha$-latrotoxin receptors. J Biol Chem 2002;277:35887-95.

[29] Valtorta F, Madeddu L, Meldolesi J, Ceccarelli B. Specific localization of the a-latrotoxin receptor in the nerve terminal plasma membrane. J Cell Biol 1984;99:124-32.

[30] Pescatori M, Grasso A. Characterization of the epitope for $4 \mathrm{C} 4.1 \mathrm{mAb}$ on $\alpha$-latrotoxin using phage display-peptide libraries: prevention of toxin-dependent ${ }^{45} \mathrm{Ca}^{2+}$ uptake in non-neuronal 
human embryonic cells transiently expressing latrophilin. Biochimie 2000;82:909-14.

[31] Cattaneo A, Grasso A. A functional domain on the $\alpha$-latrotoxin molecule, distinct from the binding site, involved in catecholamine secretion from PC12 cells: identification with monoclonal antibodies. Biochemistry 1986;25:2730-6.

[32] Graudins A, Padula M, Broady KW, Nicholson GM. Red-back spider (Latrodectus hasselti) antivenom prevents the toxicity of widow spider venoms. Ann Emerg Med 2001;37:154-60.

[33] Keegan $\mathrm{H}$. Effectiveness of Latrodectus tredecimguttatus antivenin in protecting laboratory mice against effects of intraperitoneal injections of Latrodectus mactans. Am J Trop Med Hygiene 1955;4:762-4.

[34] Muller GJ. Black and brown widow spider bites in South Africa. A series of 45 cases. South African Med J 1993;83:399-405.

[35] Daly FF, Hill RE, Bogdan GM, Dart RC. Neutralization of Latrodectus mactans and L. hesperus venom by redback spider (L. hasselti) antivenom. J Toxicol-Clin Toxicol 2001;39:119-23.

[36] Platnick NI. The world spider catalog, version 10.5. American Museum of Natural History. 2010: Online at http://research.amnh.org/entomology/spiders/catalog/index.html.

[37] Ginsborg BL, Warriner J. The isolated chick biventer cervicis nerve-muscle preparation. Brit J Pharmacol Chemother 1960;15:410-1.

[38] Chong Y, Hayes JL, Sollod B, Wen S, Wilson DT, Hains PG, et al. The $\omega$-atracotoxins: selective blockers of insect M-LVA and HVA calcium channels. Biochem Pharmacol 2007;74:62338.

[39] Volkova TM, Galkina TG, Kudelin AB, Grishin EV. [Structure of tryptic fragments of a neurotoxin from black widow spider venom]. Bioorganicheskaia Khimiia 1991;17:437-41.

[40] Aljanabi SM, Martinez I. Universal and rapid salt-extraction of high quality genomic DNA for PCR-based techniques. Nucleic acids research 1997;25:4692-3.

[41] King GF, Gentz MC, Escoubas P, Nicholson GM. A rational nomenclature for naming peptide toxins from spiders and other venomous animals. Toxicon 2008;52:264-76.

[42] Herzig V, Wood DLA, Newell F, Chaumeil P-A, Kaas Q, Binford GJ, et al. ArachnoServer 2.0, an updated online resource for spider toxin sequences and structures. Nucleic Acids Res 2011;39:D653-D7. 
[43] Wood DL, Miljenovic T, Cai S, Raven RJ, Kaas Q, Escoubas P, et al. ArachnoServer: a database of protein toxins from spiders. BMC Genomics 2009;10:375.

[44] Boeckmann B, Bairoch A, Apweiler R, Blatter MC, Estreicher A, Gasteiger E, et al. The SWISS-PROT protein knowledgebase and its supplement TrEMBL in 2003. Nucleic Acids Res 2003;31:365-70.

[45] Tzeng MC, Tian SS. Use of chick biventer cervicis muscle in the bioassay of a-latrotoxin from black widow spider venom. Toxicon 1983;21:879-81.

[46] Graudins A, Gunja N, Broady KW, Nicholson GM. Clinical and in vitro evidence for the efficacy of Australian red-back spider (Latrodectus hasselti) antivenom in the treatment of envenomation by a cupboard spider (Steatoda grossa). Toxicon 2002;40:767-75.

[47] Kiyatkin N, Dulubova I, Chekhovskaya I, Lipkin A, Grishin E. Structure of the low-molecularweight protein copurified with a-latrotoxin. Toxicon 1992;30:771-4.

[48] Grasso A. Preparation and properties of a neurotoxin purified from the venom of black widow spider (Latrodectus mactans tredecimguttatus). Biochimia et Biophysica ACTA 1976;439:406-12.

[49] Hirano H, Komatsu S, Takakura H, Sakiyama F, Tsunasawa S. Deblocking and subsequent microsequence analysis of $\mathrm{N}^{\alpha}$-blocked proteins electroblotted onto PVDF membrane. J Biochem (Tokyo) 1992;111:754-7.

[50] Hirano H, Komatsu S, Kajiwara H, Takagi Y, Tsunasawa S. Microsequence analysis of the $\mathrm{N}$-terminally blocked proteins immobilized on polyvinylidene difluoride membrane by western blotting. Electrophoresis 1993;14:839-46.

[51] Bork P. Hundreds of ankyrin-like repeats in functionally diverse proteins: mobile modules that cross phyla horizontally? Proteins 1993;17:363-74.

[52] Michaely P, Bennett V. The ANK repeat: a ubiquitous motif involved in macromolecular recognition. Trends Cell Biol 1992;2:127-9.

[53] Mosavi LK, Minor DL, Jr., Peng ZY. Consensus-derived structural determinants of the ankyrin repeat motif. Proc Nat Acad Sci U S A 2002;99:16029-34.

[54] Rohou A, Nield J, Ushkaryov YA. Insecticidal toxins from black widow spider venom. Toxicon 2007;49:531-49.

[55] Ichtchenko K, Khvotchev M, Kiyatkin N, Simpson L, Sugita S, Sudhof TC. a-Latrotoxin 
action probed with recombinant toxin: receptors recruit a-latrotoxin but do not transduce an exocytotic signal. EMBO J 1998;17:6188-99.

[56] Theakston R, Warrell DA. Antivenoms: a list of hyperimmune sera currently available for the treatment of envenoming by bites and stings. Toxicon 1991;29:1419.

[57] Bugli F, Graffeo R, Sterbini FP, Torelli R, Masucci L, Sali M, et al. Monoclonal antibody fragment from combinatorial phage display library neutralizes a-latrotoxin activity and abolishes black widow spider venom lethality, in mice. Toxicon 2008;51:547-54.

[58] Pashkov V, Grico N, Tsurupa G, Storchak L, Shatursky O, Himmerlreich N, et al. Monoclonal antibodies can uncouple the main a-latrotoxin effects: toxin-induced $\mathrm{Ca}^{2+}$ influx and stimulated neurotransmitter release. Neuroscience 1993;56:695-701.

Table 1: Sequence similarities for latrotoxins from $L$. hasselti, L. hesperus, and L. mactans determined by MS-MS

\begin{tabular}{llcc}
\hline \multirow{2}{*}{$\begin{array}{l}\text { Known sequence } \\
\text { (L. tredecimguttatus) }\end{array}$} & & \multicolumn{2}{c}{ MS-MS Sequence comparison } \\
\cline { 3 - 4 } & L. hasselti & $\begin{array}{c}\text { \% Identity } \\
\text { (identities/total) }\end{array}$ & $\begin{array}{c}\text { \% Similarity } \\
\text { (similarities/total) }\end{array}$ \\
\hline \multirow{2}{*}{-latrotoxin $^{1}$} & L. hesperus & $100(54 / 54)$ & $100(54 / 54)$ \\
& L. mactans & $94.6(148 / 150)$ & $98(147 / 150)$ \\
\hline -latroinsectotoxin $^{2}$ & L. hasselti & $100(151 / 151)$ & $100(151 / 151)$ \\
\hline \multirow{2}{*}{-latroinsectotoxin $^{3}$} & L. hasselti & $88.7(63 / 71)$ & $91.5(65 / 71)$ \\
& L. hesperus & $91.2(133 / 148)$ & $93.2(135 / 148)$ \\
\hline
\end{tabular}

Similarities and identities are based on comparisons with the known sequences of L. tredecimguttatus latrotoxins. ${ }^{1}$ Kiyatkin et al., 1990, ${ }^{2}$ Kiyatkin et al., 1993, ${ }^{3}$ Dulubova et al., 1996. 
Figure legends

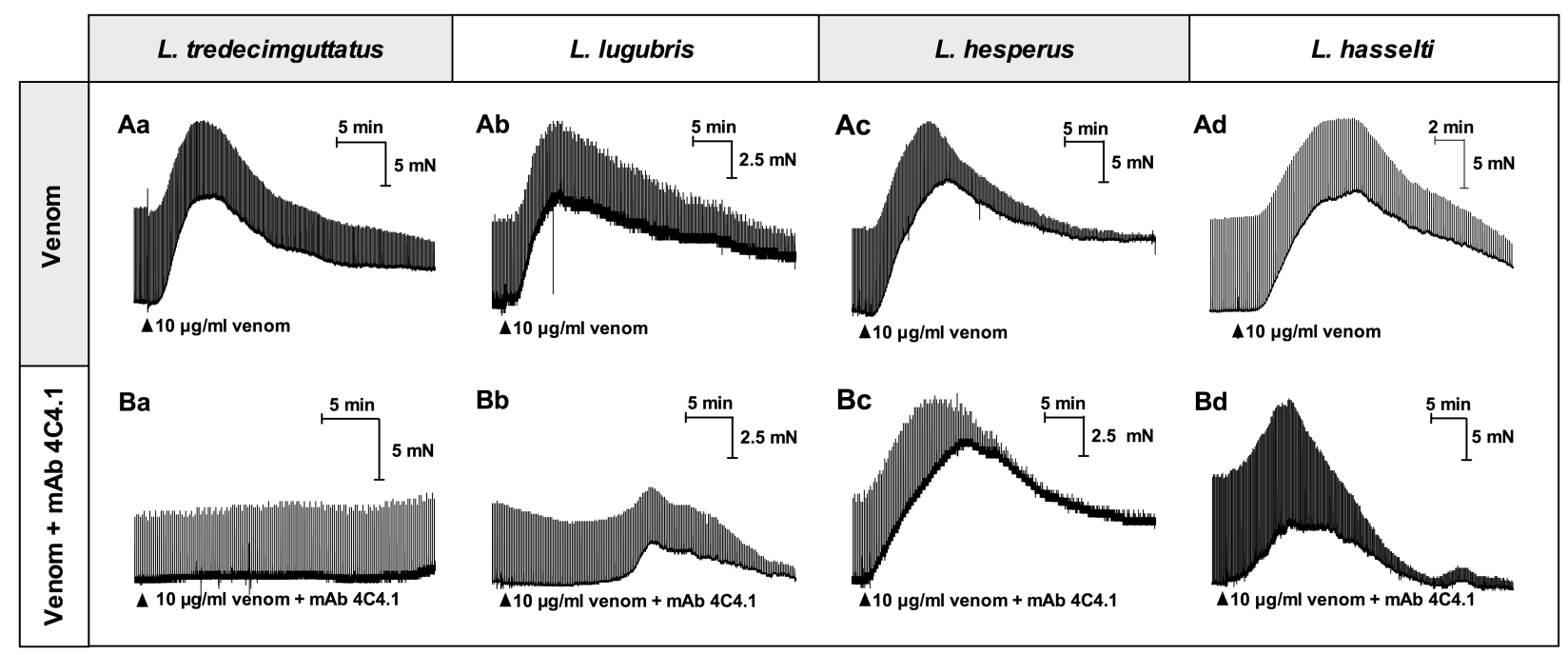

Fig. 1. Lack of cross-reactivity of the monoclonal antibody $4 \mathrm{C} 4.1$ as judged by it ability to prevent the neurotoxicity of Latrodectus spp. venoms in the isolated CBCNM preparation. (A) Typical effects of $11 \mu \mathrm{g} / \mathrm{ml}$ L. tredecimguttatus venom (a), $10 \mu \mathrm{g} / \mathrm{ml}$ L. mactans (b), $11 \mu \mathrm{g} / \mathrm{ml}$ L. hesperus (c), and $11 \mu \mathrm{g} / \mathrm{ml} \mathrm{L}$. hasselti (d) venom on indirectly-stimulated twitch contractions in the CBCNM preparation. (B) Typical effects of the same venoms recorded after a 5 min pre-incubation of venom with $1 \mathrm{ml}(5 \mu \mathrm{g})$ of the monoclonal antibody $4 \mathrm{C} 4.1$. 

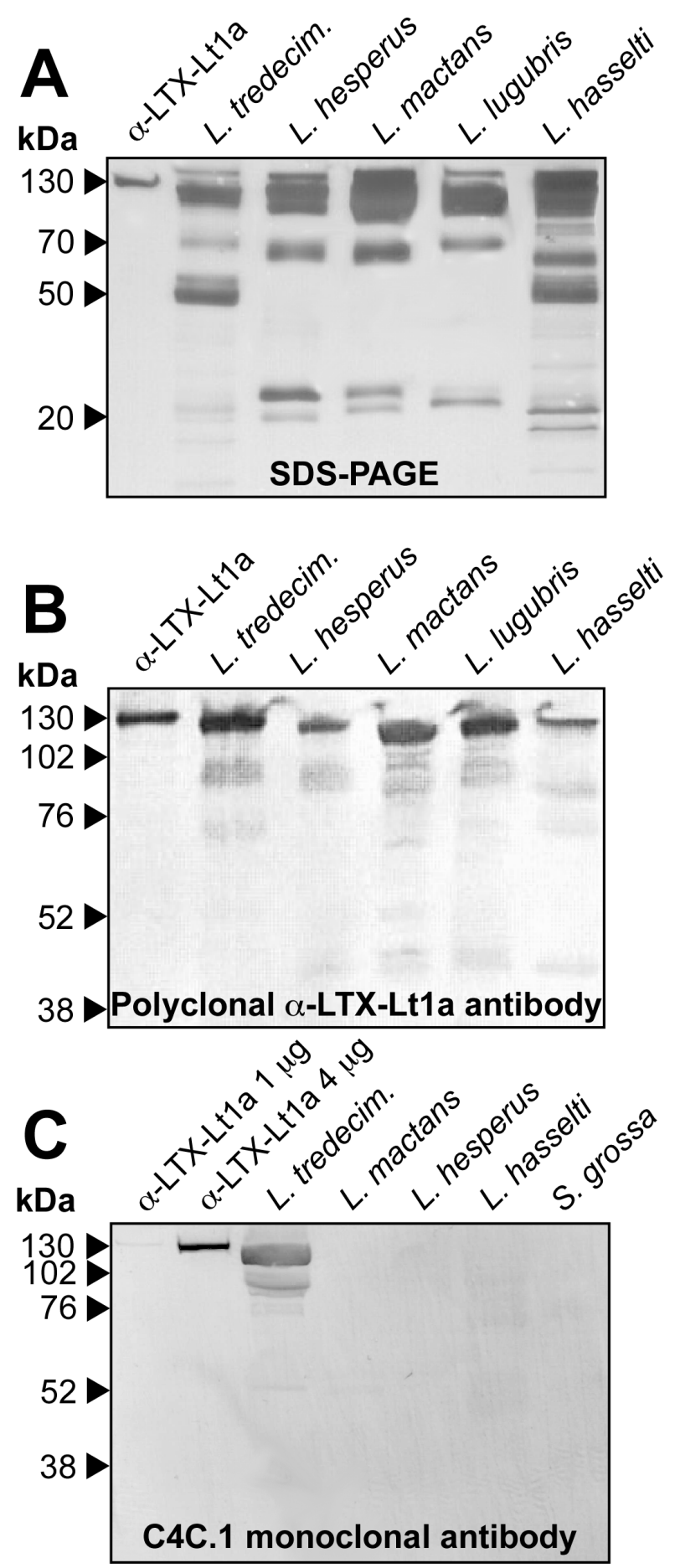

Fig. 2. SDS-PAGE gel and Western blots of $\alpha$-LTX-Lt1a and theridiid spider venoms. (A) SDSPAGE gel run under reducing conditions showing $\alpha$-LTX-Lt1a and protein components of Latrodectus spp. venoms. Note a consistent protein band in the venom lanes at $\sim 130 \mathrm{kDa}$, consistent with $\alpha$-LTX-Lt1a. (B-C) Western blot analyses of $\alpha$-LTX-Lt1a and theridiid venoms employing polyvalent anti- $\alpha-L T X$ antibody (B) and the monoclonal antibody $4 C 4.1$ (C) as the primary antibodies. Note that purified $\alpha$-LTX-Lt1a as well as the $130 \mathrm{kDa}$ protein band in L. tredecimguttatus venom are recognized by both antibodies. 


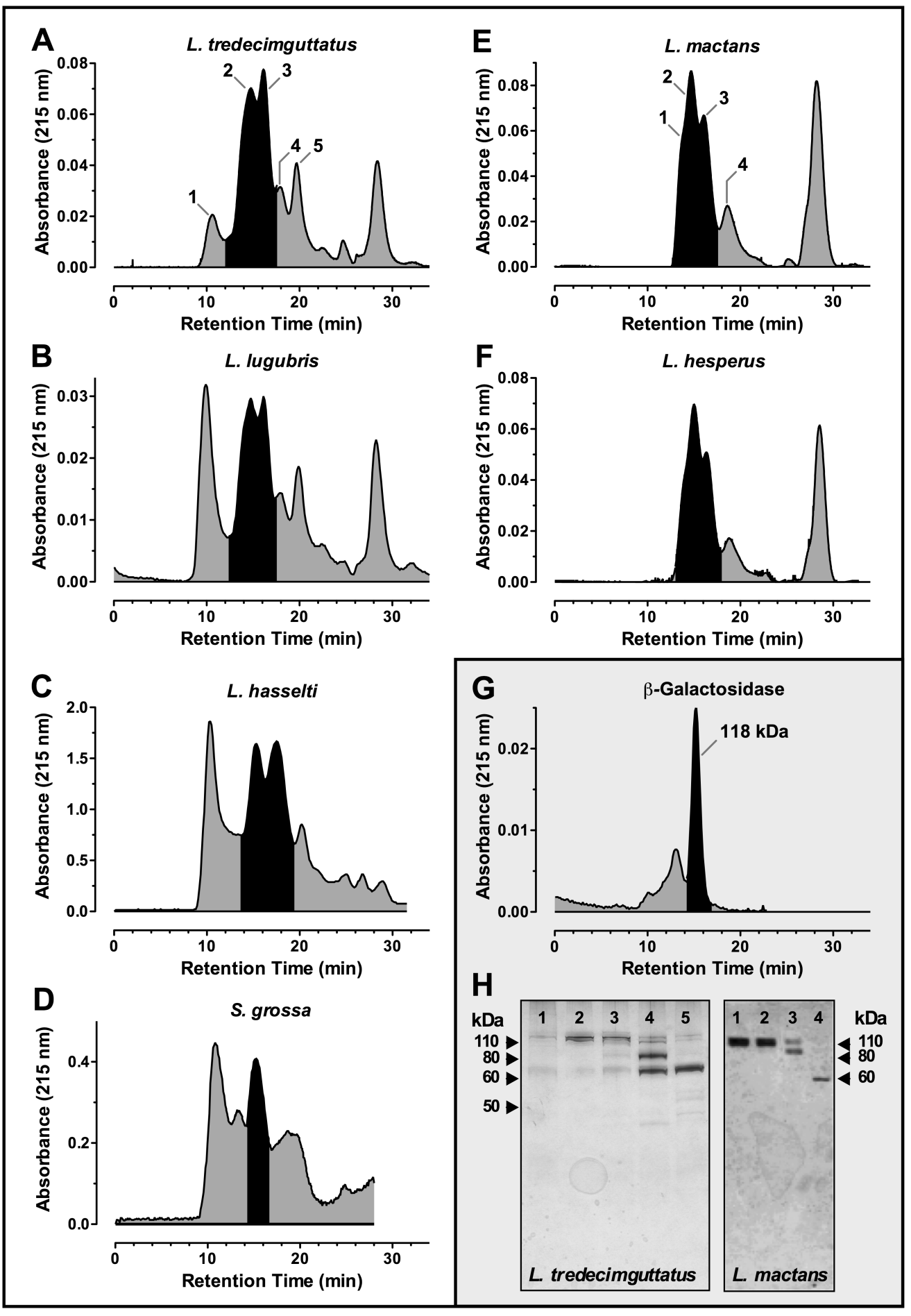

Fig. 3. Chromatographic fractionation of theridiid spider venoms. (A-F) Size-exclusion FPLC chromatograms of Latrodectus and S. grossa venoms. The black shaded portion of the chromatogram indicates fractions with vertebrate activity in the CBCNM preparation. (G) Chromatogram obtained for the molecular mass marker $\beta$-galactosidase (118 kDa) under the same elution conditions $(H)$ SDS-PAGE gels run under reducing conditions of $L$. tredecimguttatus and L. mactans size exclusion fractions. Lanes correspond to the peaks marked in panels $A$ and $E$, respectively. 


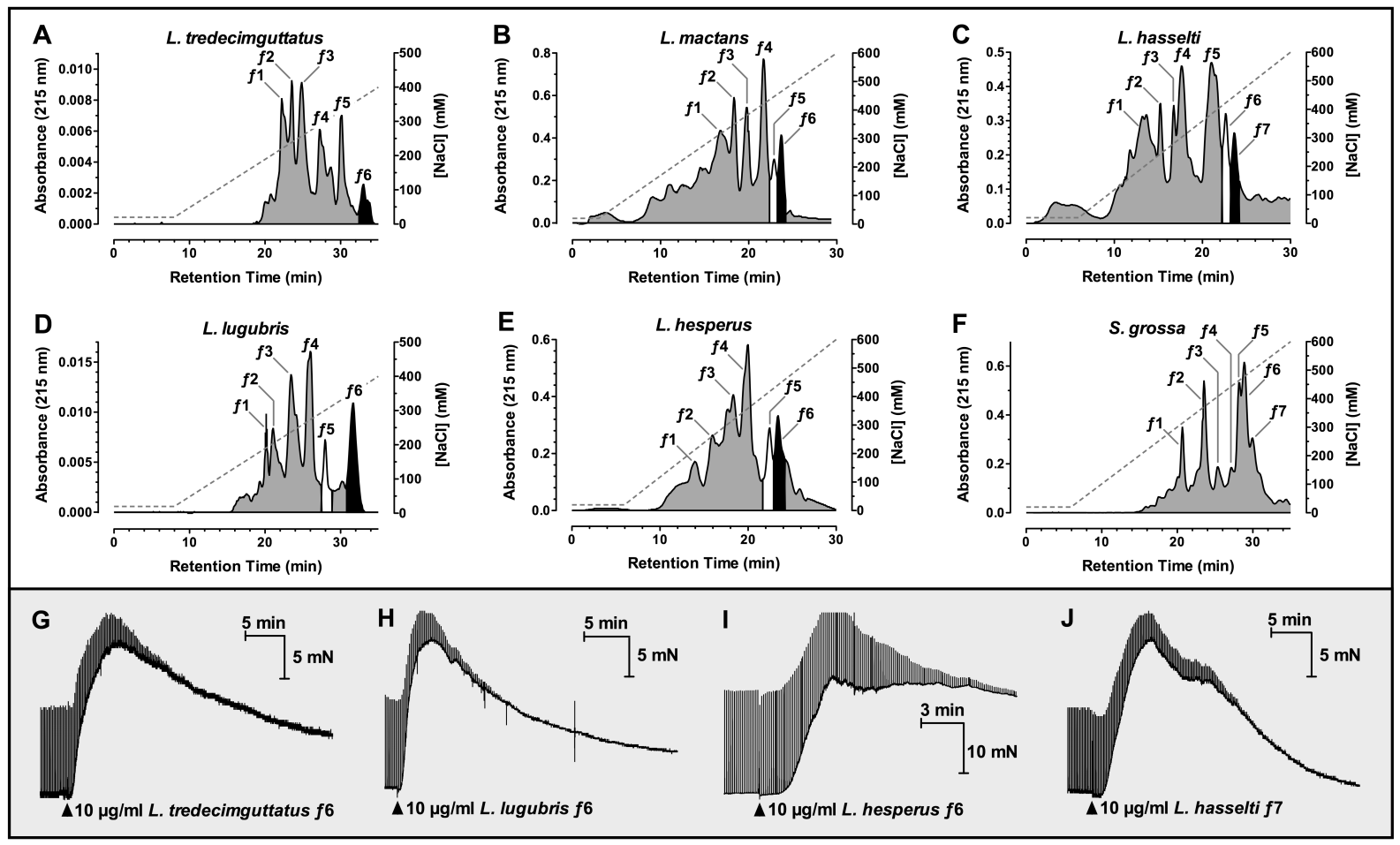

Fig. 4. Anion-exchange FPLC separation of vertebrate-toxic intermediate-mass protein fractions from theridiid spider venoms. (A-F) Anion-exchange FPLC chromatograms recorded at $215 \mathrm{~nm}$ (left-hand ordinate axis) using a linear $\mathrm{NaCl}$ gradient (right-hand ordinate axis). The final eluting peaks shaded in black ( $f 6$ or $f 7$ ) indicate fractions with vertebrate toxicity. The preceding peaks shaded in white ( $f 5$ or $f 6$ ) indicate fractions with insect toxicity. Note the similarity in chromatographic profiles and the overlap of adjacent peaks. The anion-exchange profile obtained for S. grossa venom is markedly different to that obtained for the Latrodectus venoms. Additionally, S. grossa fractions did not exhibit any activity in the chick bioassay. (G-J) Effect of $10 \mu \mathrm{g} / \mathrm{ml} f 6$ or $f 7$ fractions from anion-exchange FPLC on indirectly-stimulated twitch contractions in the CBCNM preparation. 
A

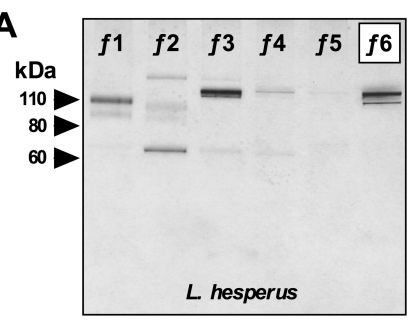

D

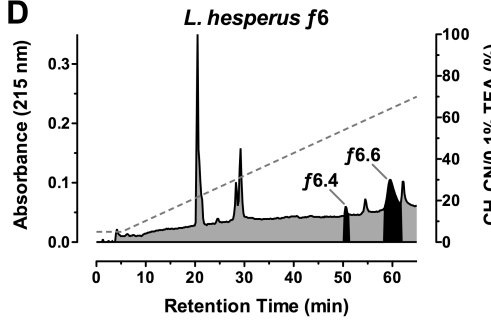

B
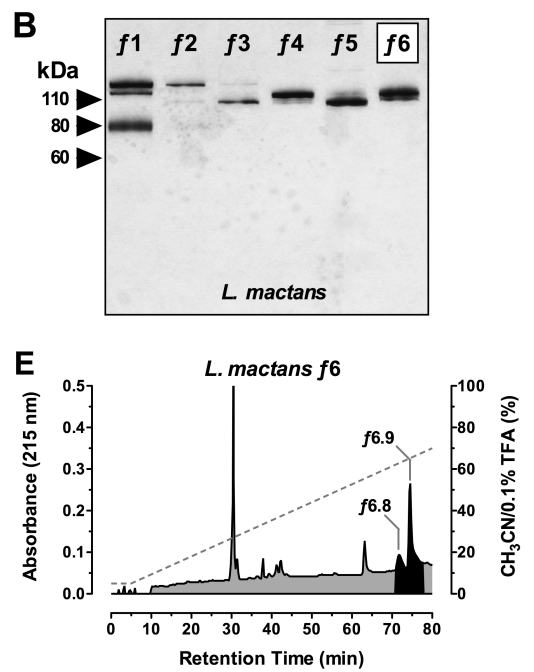
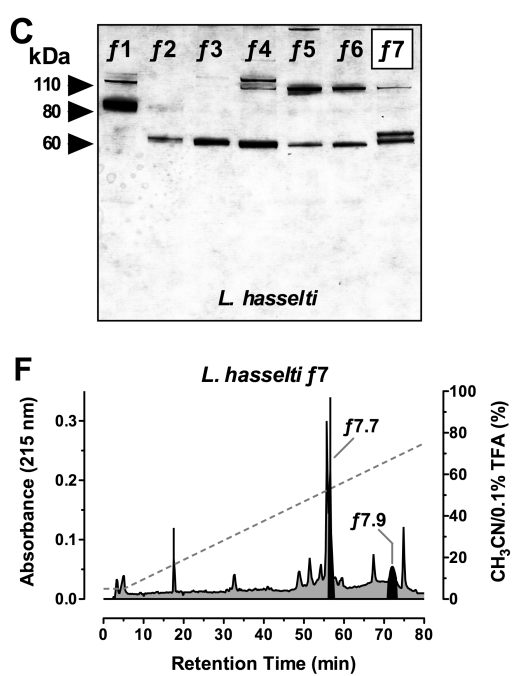

Fig. 5. Electrophoretic and reverse-phase separation of vertebrate-toxic anion-exchange FPLC fractions. (A-C) Typical SDS-PAGE gels run under reducing conditions of anion-exchange FPLC fractions. Lanes correspond to the peaks marked in Fig. 4. (D-F) Analytical C18 RP-HPLC chromatograms obtained for vertebrate-toxic anion-exchange FPLC fractions ( $f 6$ or $f 7$ ) recorded at $215 \mathrm{~nm}$ (left-hand $y$-axes) using a linear $0.1 \%$ TFA/acetonitrile gradient (right-hand ordinate axes). Peaks highlighted in black were submitted for MS-MS analysis. 


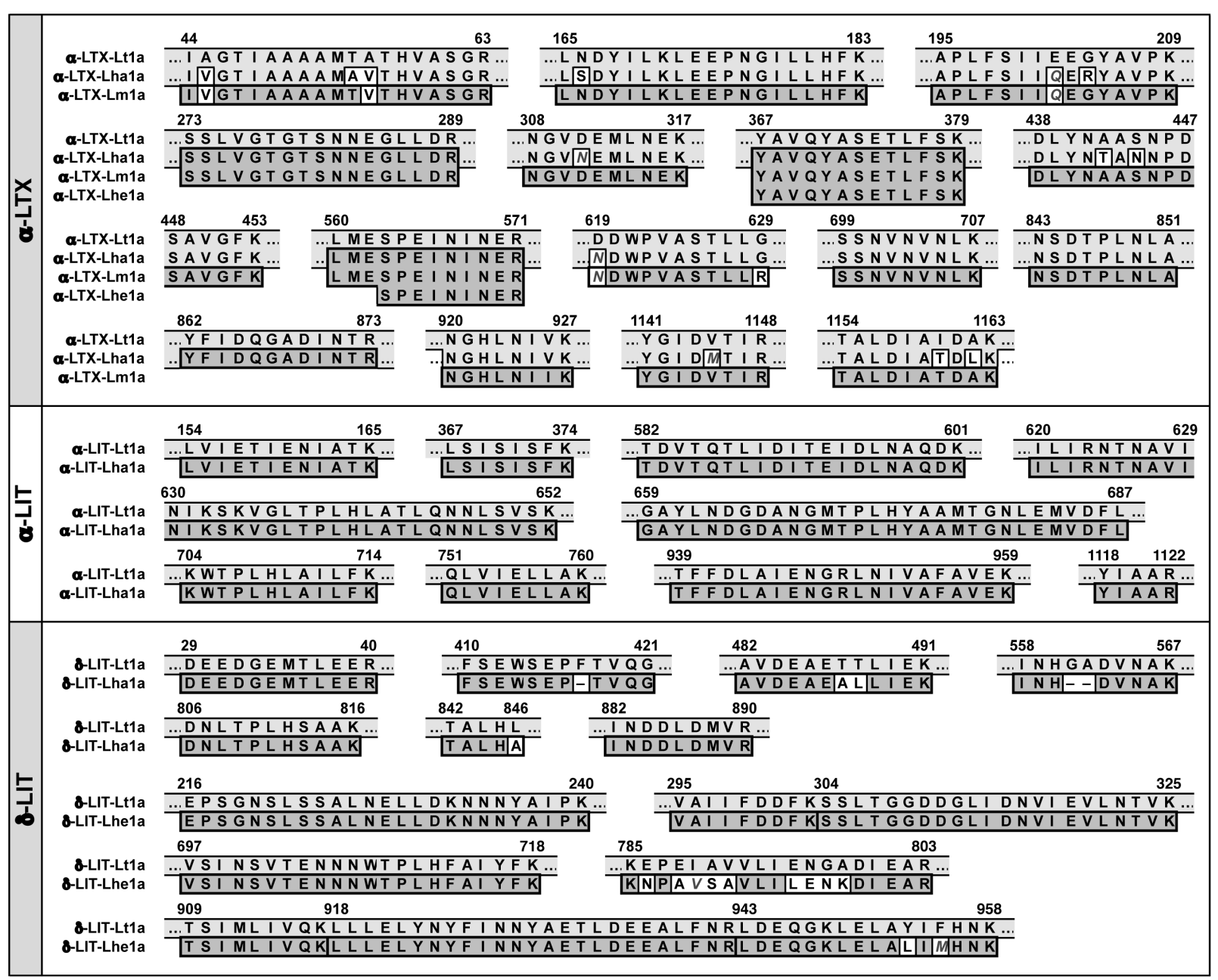

Fig. 6. Comparison of the MS-MS peptide sequences from RP-HPLC separation of the vertebratetoxic anion-exchange fraction from Latrodectus spp. venoms. The amino acid sequences were determined by MS-MS sequencing following overnight (16 $\mathrm{h}$ ) digestion with trypsin. Sequences are aligned against the known sequences of $\alpha$-LTX-Lt1a, $\alpha$-LIT-Lt1a and $\delta$-LIT-Lt1a from L. tredecimguttatus (light gray) [8-10]. Identities are boxed in dark gray, conservative substitutions are in gray italic text. Isoleucine and leucine residues were assigned from the known sequences. 
a-LTX-Lha1a
a-LTX-LHa

a-LTX-Ltala

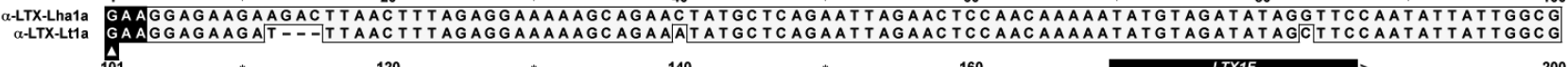

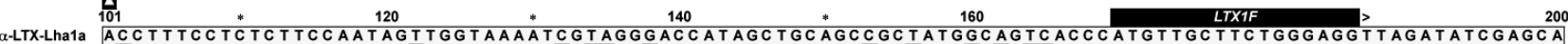

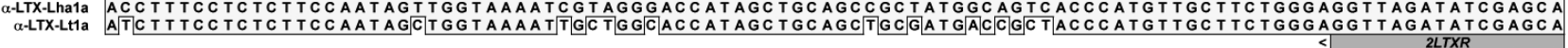

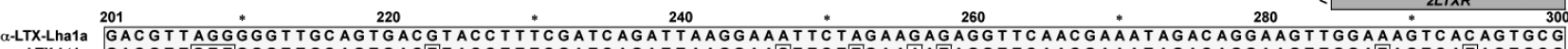

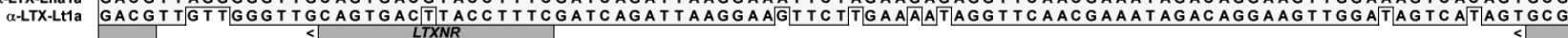
301

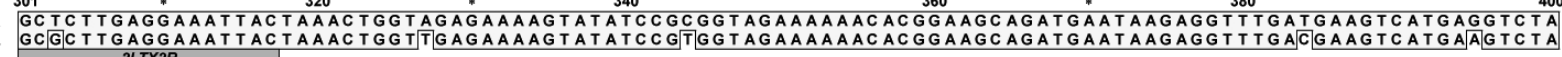
$401 \quad 217 \times 2 R$

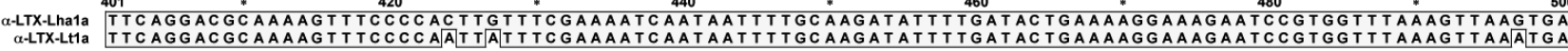

a-LTX-Lha1a 50101000

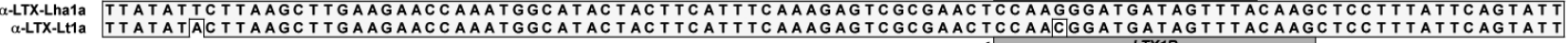

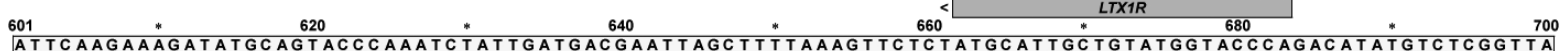

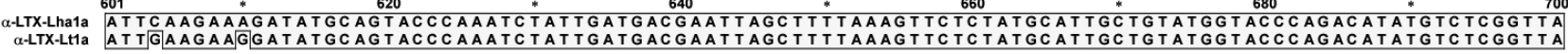

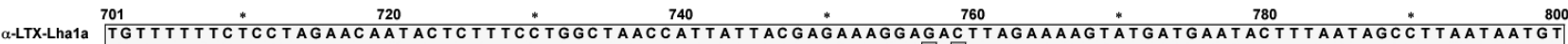

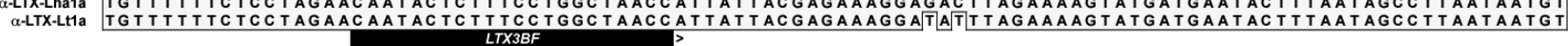
8010

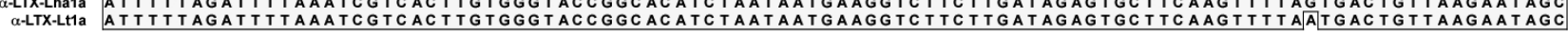

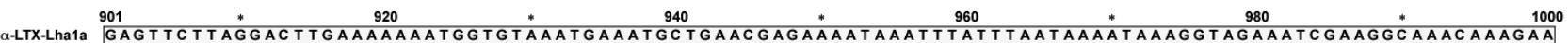

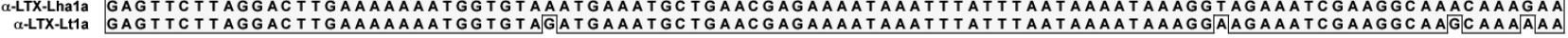

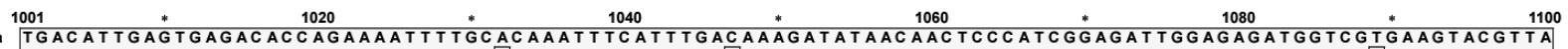

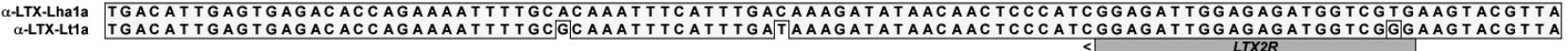

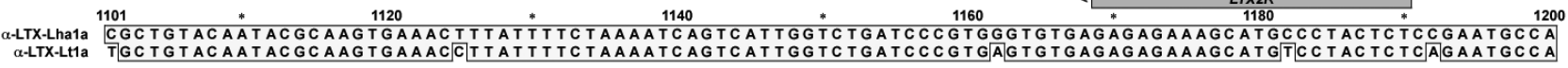
12010001200

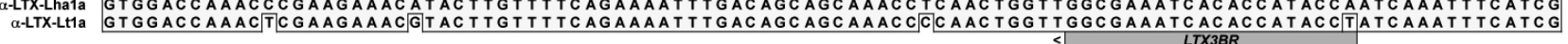

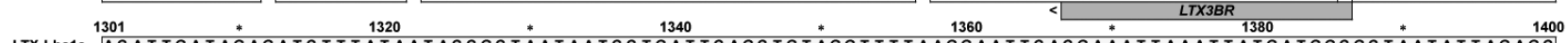

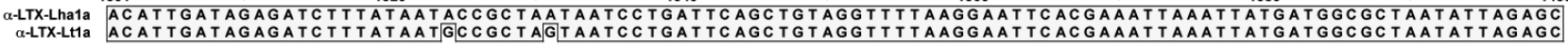

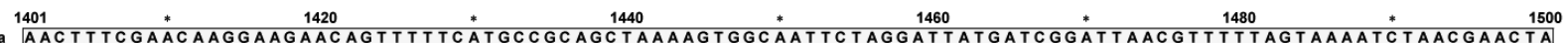

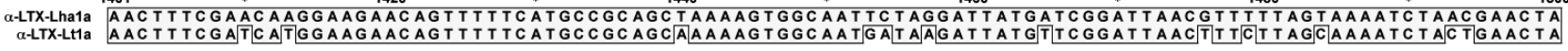

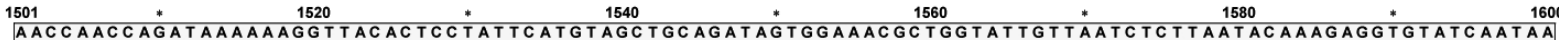

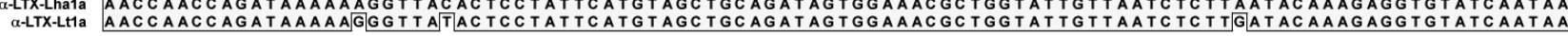

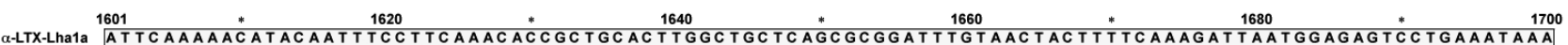
a-LTX-Lta ATTCAAAAACATACCATTTCCTTCAAACCLCGTTGCACTTGGCTGCTCAGCGCGGATTTTAACTACTTTTCAAGATTAATGGAAAGCLCTGAAATAAA

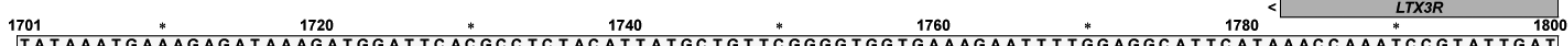
a-LTX-Lha1a T17 TATAAATGAAAGAGATAAAGATGGAT TCACGCC TC TACAT TATGC TGT TCGGGGTGG TGAAAGAAT T T TGGAGGCAT TCATAAACCAAATCCGTAT TGA

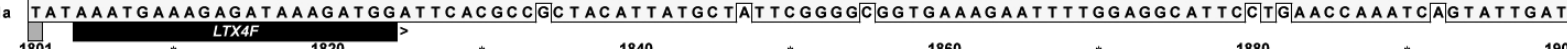

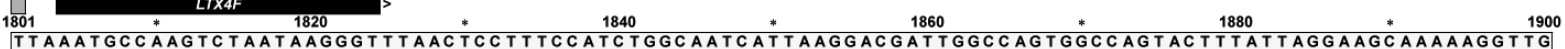

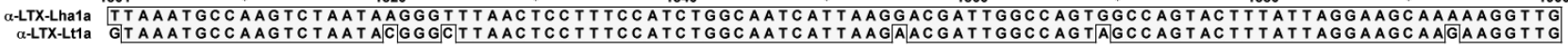

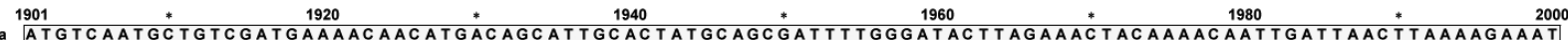

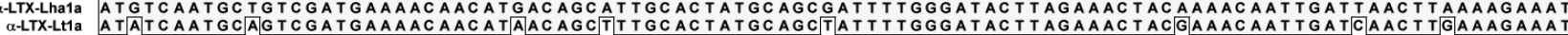




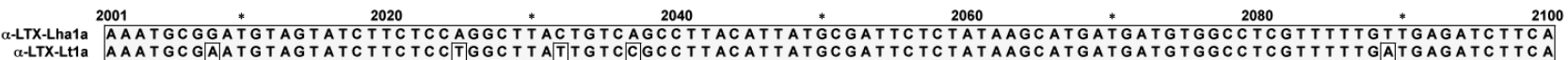

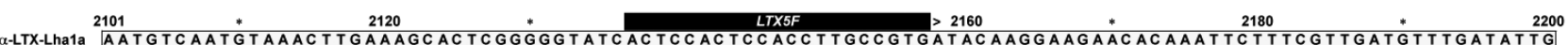
a-LTX-Lha1a AATGTCAATGTAAACT TGAAAGCACTCGGGGGTATCACTCCACTCCTXGCT

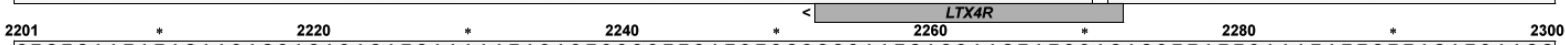
a-LTX-Lha1a GTG TGAATATAGAACAGCAGACAGATGAAAAATACAC TCCCCTTCATC TCGCGGCAATGAGCAAGTATCCAGAGC TTAT TCAAATAT TG TTAGATCAAGG a-LTX-Lt1a GTGTGAATATAGAACAGAAGACAGATGAAAAATATACGCCCCTTCATCTCGCGGCAATGAGTAAGTATCCAGAGCTTATTCAAATATTGTTAGATCAAGG

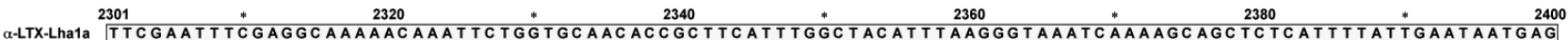

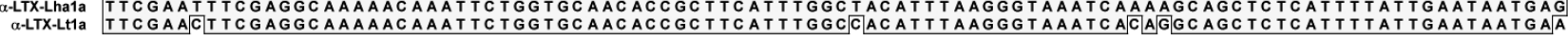

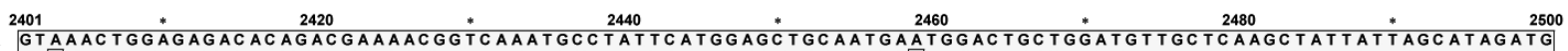
a-LTX-Lt1a GTGAACTGGAGAGACACAGACGAAAACGGTCAAATGCCTATTCATGGAGCTGCAATGACTGGACTGCTGGATGTTGCTCAAGCTATTATTAGCATAGATG

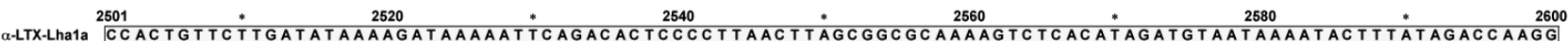
a-LTX-Lha1 CCACTGTTCTTGATATAAAAGATAAAAATTCAGACACTCCCCTTAACTTAGCGGCGCAAAAGTC TCACATAGATG TAATAAAATACTTTATAGACCAAGG

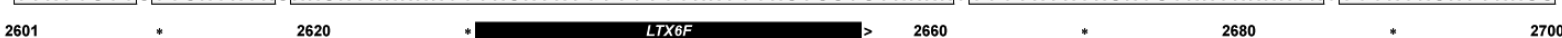

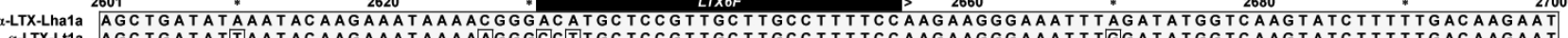

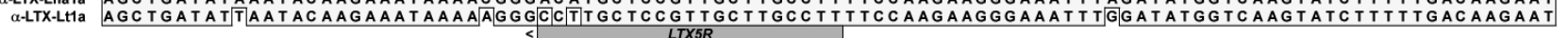
$2701 \quad 2720 \quad 27200$ a-LTX-LHa GCTAATGTTATATCGCTGACAATGATGGTATGAATTTTTTTACTATGCAGTTCAAAACGGTCATTTGAATATAGTTAAATACGCTATGTCAGAAAAAG

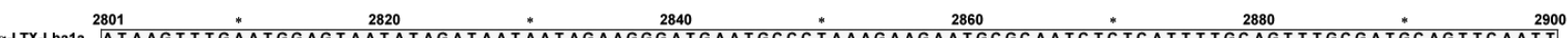
-LTX-Lha1a ATAAGTTTGAATGGAGTAATATAGATAATAATAGAAGGGATGAATGCCC TAAAGAAGAATGCGCAATC TCTCATT TTGCAGTTTGCGATGCAGTTCAATT

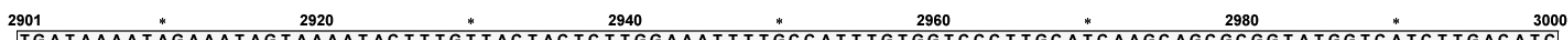
a-LTX-Lha1 ThGATAAAATAGAAATAGTAAAATACTTTGTTAC TACTCTTGGAAATTTTGCCATTTGTGGTCCCTTGCATCAAGCAGCGCGGTATGGTCATCTTGACATC a-LTX-LHa TGATAGAATAGAAATAGTAAAATACTTTGTTGGIACTCTTGGAAATTTGCCATTTGTGGTCCCCTGCATCAAGCAGCGCGGTACGGTCATCTTGACATA $30013013040 \quad * 310030$ a-LTX-Lt1a GTGAAATATCTGGTCGAAGAAGAATTICTGAGTGTGGATGGAAGCAAAACTGATACTCCCTTGTGTTACGCTTCTGAAAATGGACATTTTACGGTTGTTC

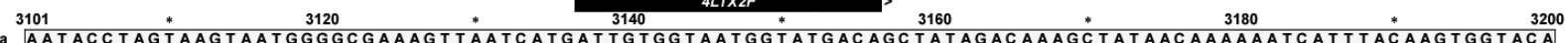

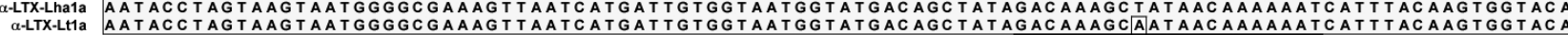

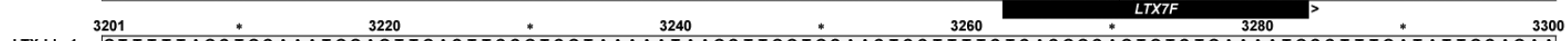
a-LTX-Lha1 320 TT TT AGC TGCAAATGGAGTTGACTTCCGTCGTAAAAATAAGCTTGGTGCAACTCCTTTTCTCACGGCAGTGTCTGAAAATGCCT TTGATATTGCAGAA $\begin{array}{cccc}3301 & 3320 & 3340 & 3360\end{array}$

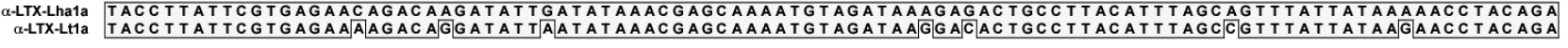

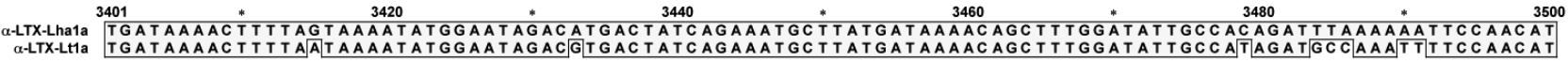

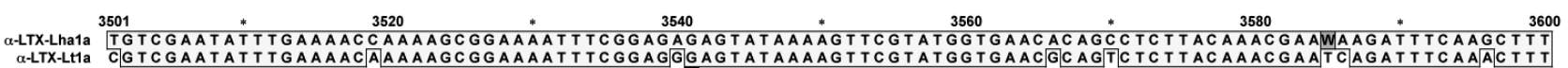
\begin{tabular}{lllllll}
3601 & $*$ & 3620 & $*$ & 3640 & $*$ & 3680 \\
\hline
\end{tabular} a-LTX-Lha1a ATAGATGGTAAGAACATCGAACAWGACCATCCCCAATT TATAAATGCAGATAATGAGTCGTCTCAGT TGT TT TCGGACACAGC TTC TAACATTGACGTCA a-LTX-Lt1a ATAGATCGTAAGAACATCGAACATGGACATCCACTATTTATAAATGCAGATAATGAGTCTTCTGAGTTGTTTTCGAAAACAGCTTCCAACATTGACGTTA $3701 \quad 370 \quad 3740 \quad 360$

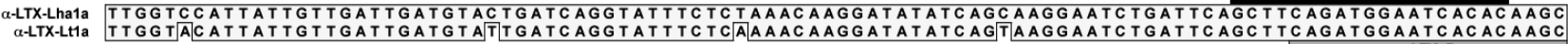

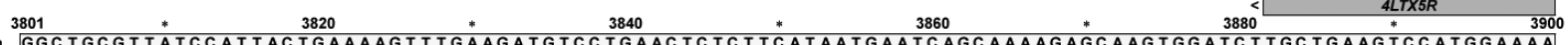
a-LTX-LL1a CGCTGCGTTATCCATTACTGAAAAGT TCGAAGATGTCTTGAACTCTCTTCATAATGAATCAGCAAAAGAGCAGGTGGATCTTGCTGAAGTCCATGGGAAA

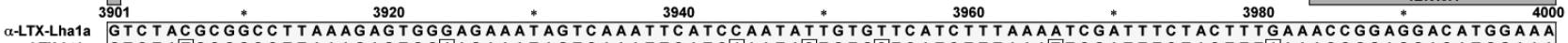

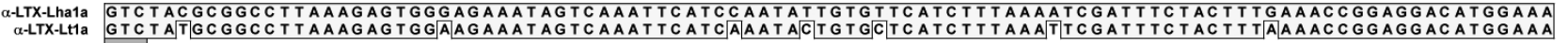

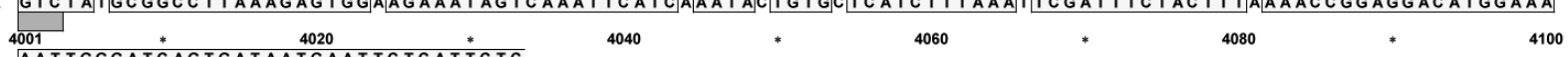

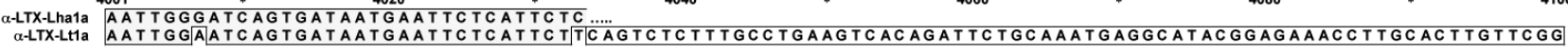

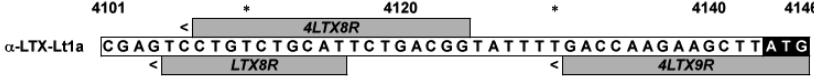

Fig. 7. Alignment of the nucleotide sequence of $\alpha-L T X-L h 1 a$ with the known sequence of $\alpha-L T X-$ Lt1a from $L$. tredecimguttatus. The $\alpha$-LTX-Lh1a sequence was determined from a gDNA library created from female $L$. hasselti venom glands. Identities are boxed in light gray. The two upward arrowheads indicate the $\mathrm{N}$-terminus (AAG) and $\mathrm{C}$-terminus (GAG) of the mature toxin based on sequence homology with $\alpha$-LTX-Lt1a. Labels above and below the sequences indicate the forward ('F', black) and reverse ('R', gray) primers used for PCR. The W at 3585 and 3624 indicates a nucleotide base of either $\mathrm{A}$ or $\mathrm{T}$. 


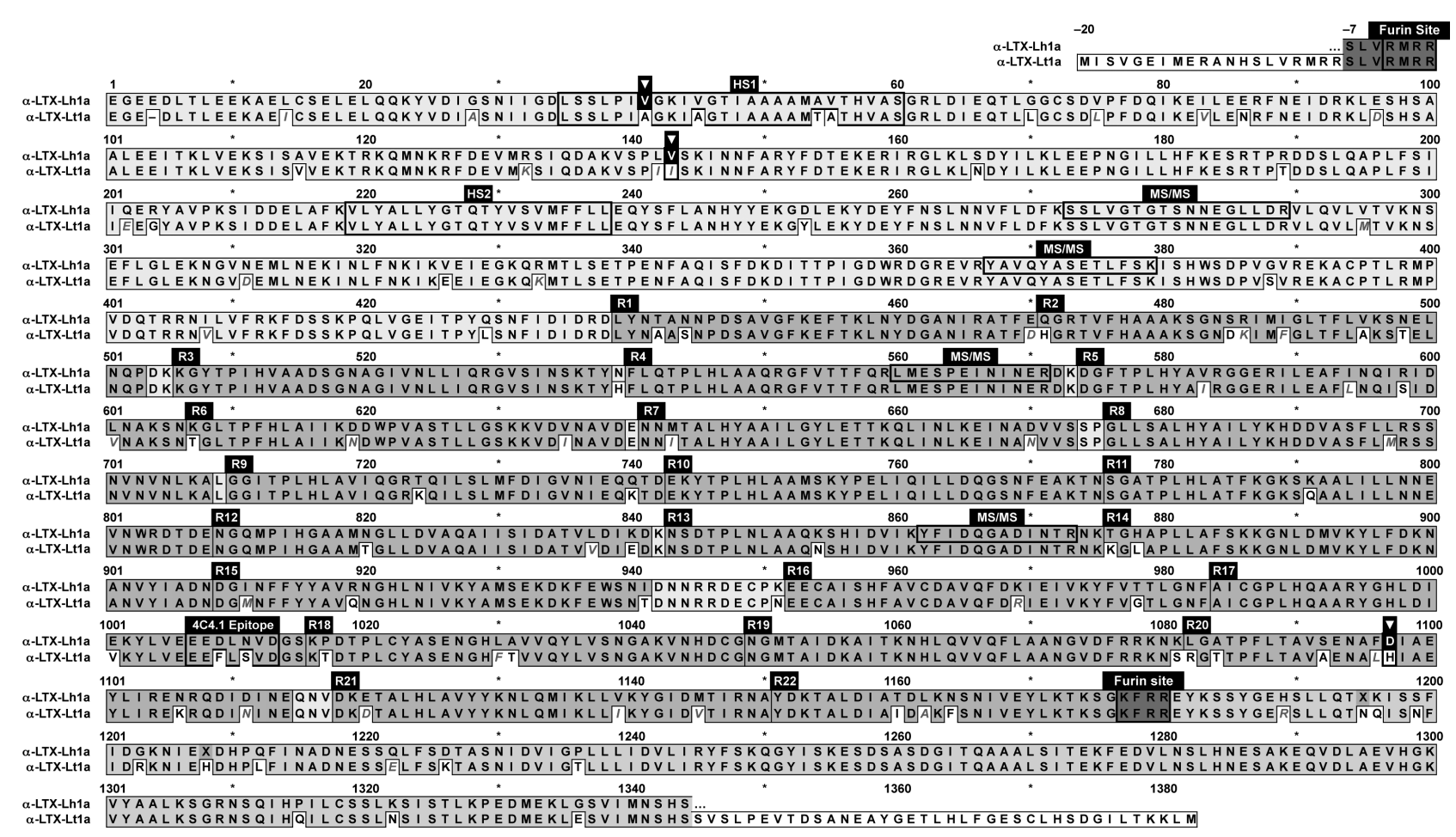

Fig. 8. Comparison of the deduced amino acid sequences of $\alpha-L T X-L h 1 a$ with $\alpha-L T X-L t 1 a$. Identities are boxed in gray, conservative substitutions are in gray italic text. Proteins are numbered from the $\mathrm{N}$-terminus of the mature $\alpha$-LTX-Lh1a based on homology with $\alpha$-LTX-Lt1a. Note insertion of a glutamate (E) residue at position 4 in $\alpha$-LTX-Lh1a. The location of the conserved furin-like endopeptidase cleavage sites for the signal sequence $(-20$ to 0$)$ and C-terminal propeptide (1281-1382) [11] are highlighted and delineate the mature toxin. The three residues marked with arrowheads denote residues in $\alpha$-LTX-Lh1a that are identical to a variant of $\alpha$-LTX-Lt1a (AAC78471 in EMBL-Bank CDS). Sequences marked 'MS-MS' denote residues also sequenced by MS-MS analysis. Positions of the 22 ankyrin-like imperfect repeats (R1-R22), hydrophobic segments (HS1 and HS2, [10]) and monoclonal antibody 4C4.1 binding epitope (residues 1007-1113; [30]) are marked above the sequences. Residue marked ' $X$ ' in the C-terminal propeptide region indicate unknown residues due to uncertainties ( $A$ or $T$ ) in the one of the bases in the corresponding nucleotide triplet: position 1208 is $N$ (corresponding residue in $\alpha$ LTX-Lt1a) or K while position 1195 is $\mathrm{H}$ (corresponding residue in $\alpha$-LTX-Lt1a) or $\mathrm{Q}$. 


\section{SUPPLEMENTARY FIGURE LEGENDS}

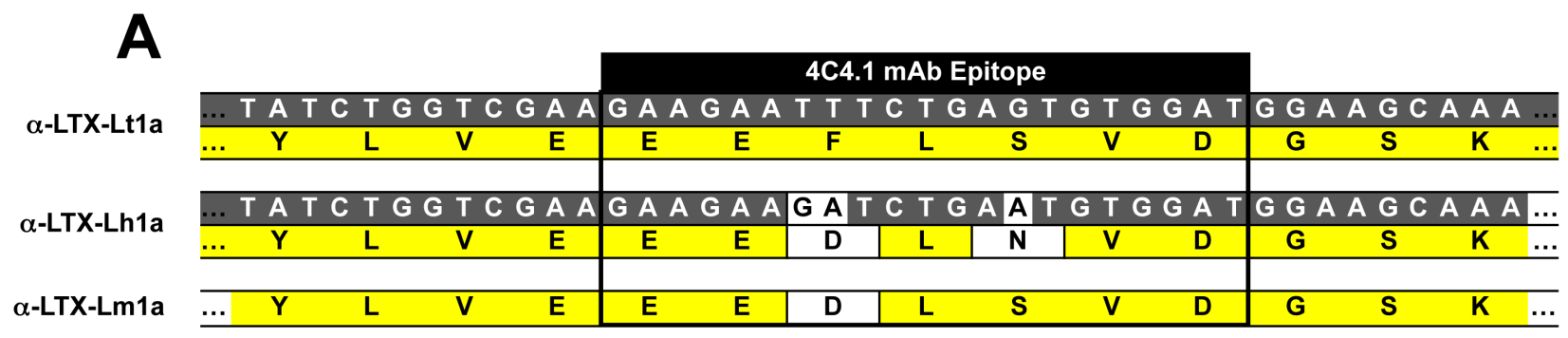

B

LMWP L. tredecimguttatus f6 LMWP L. tredecimguttatus f6 LMWP L. lugubris LMWP2 L. tredecimguttatus

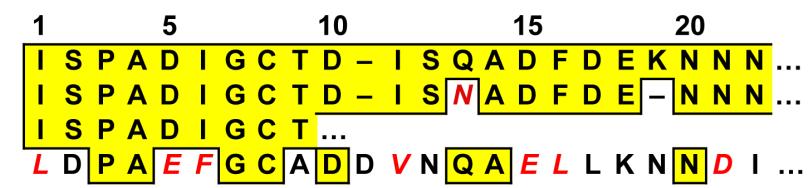

\begin{tabular}{|c|c|}
\hline$\%$ Identity & $\%$ Similarity \\
\hline 100 & 100 \\
91 & 95 \\
100 & 100 \\
36 & 68
\end{tabular}

Fig. 1S. Sequence comparisons of (A) $\alpha$-LTX-associated low molecular weight proteins, and (B) the binding epitope on $\alpha-\mathrm{LTX}$ for monoclonal antibody 4C4.1. Identities are boxed in gray, conservative substitutions are in gray italic text. (A) Alignment of the $\mathrm{N}$-terminal partial sequences of two novel LMWPs from the anion-exchange FPLC $f 6$ of $L$. lugubris and L. tredecimguttatus venom with known LMWPs. The two novel sequences were determined by Edman degradation. Note that LMWP from $L$. lugubris is $100 \%$ identical to the known sequence of LMWP from $L$. tredecimguttatus. (B) Alignment of the binding epitope for monoclonal antibody 4C4.1 on $\alpha-L T X$ from $L$. tredecimguttatus with the corresponding sites in $L$. hasselti and $L$. mactans. The binding epitope for $L$. mactans was determined from MS-MS sequencing. 
A

Ankyrin repeat consensus sequence

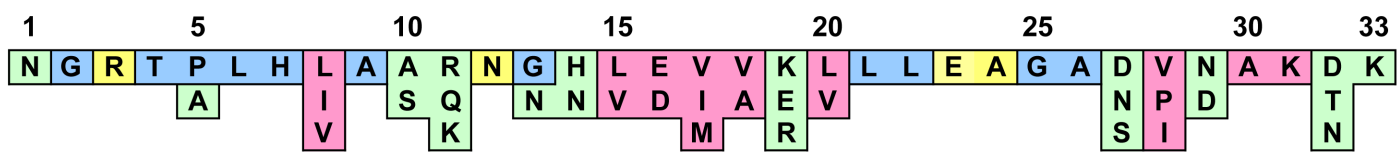

Well Conserved (>50\%)

Semi conserved (same properties)

Semi conserved (different properties)

\section{Non conserved}

B

$\alpha$-LTX-Lh1a Ankyrin-like repeats

\begin{tabular}{llllll|l|l|l}
1 & 5 & 10 & 15 & 20 & 25 & 30 & 33 & $\%$ Similarity
\end{tabular}

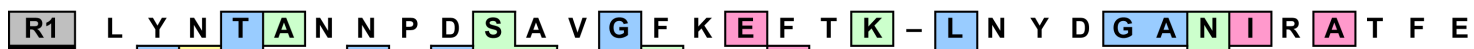

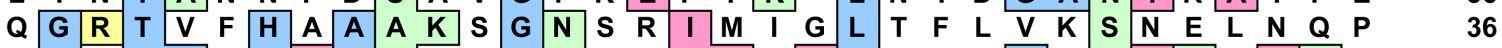

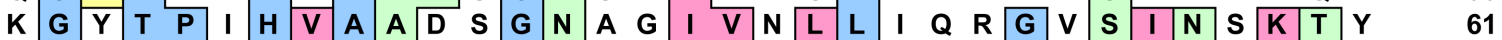

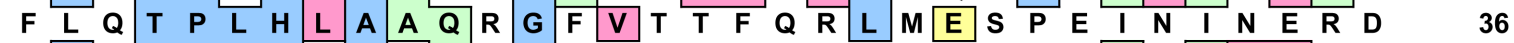

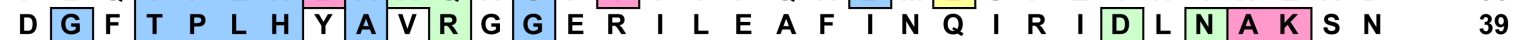

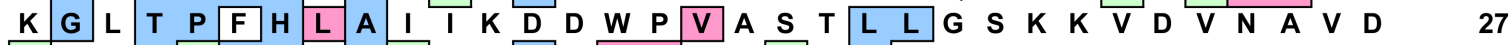
\begin{tabular}{|lllllllllllll|l|l|lllll|l|l|l|llllllllllllll}
\hline N & N & M & T & A & L & H & Y & A & A & I & L & G & Y & L & E & T & T & K & Q & L & I & N & L & K & E & I & N & A & D & V & V & S & & 39 \\
\hline
\end{tabular}

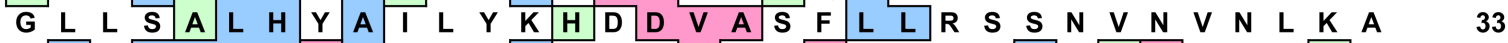

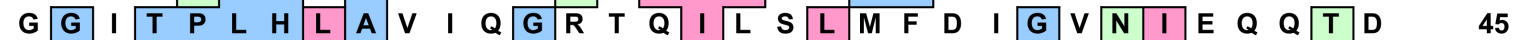

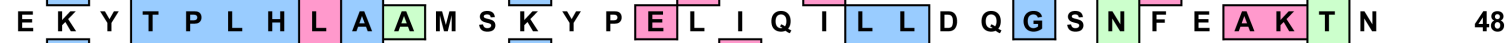

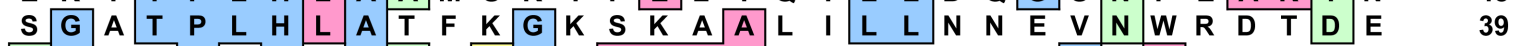

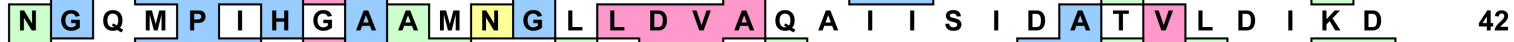

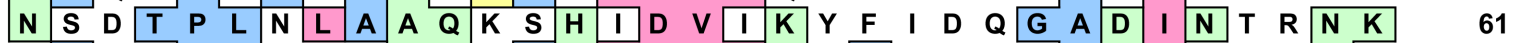

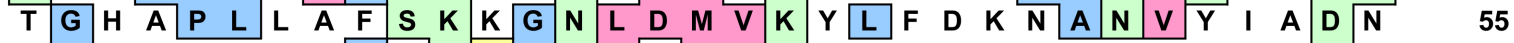

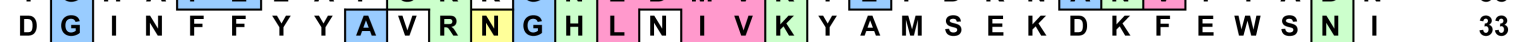

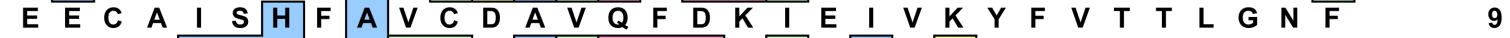

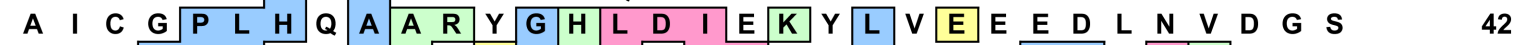

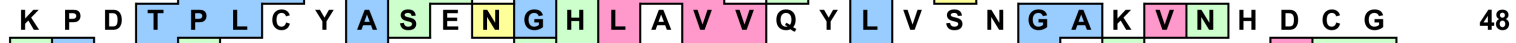

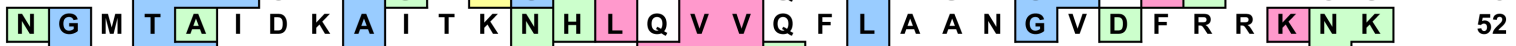

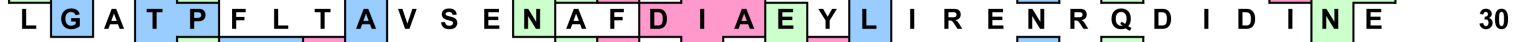

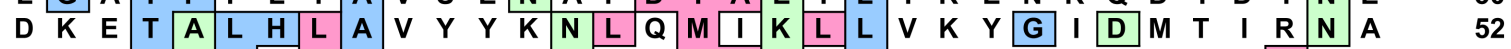

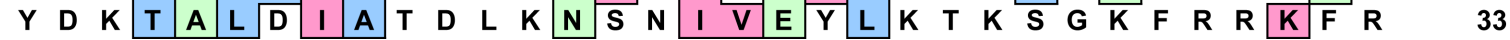

Fig. 2S. Identification of ankyrin-like repeats in $\alpha$-LTX-Lh1a. (A) Consensus sequence for the 33residue ankyrin repeat determined from a large number of proteins with ankyrin repeats. Each position is color-coded according to the level of conservation described previously $[52,53]$. Blue indicates a residue that is normally present $>50 \%$ of the time in ankyrin repeats in a wide variety of proteins. Positions are coloured red if residues occur with a higher frequency than any other residues and residues had the same physicochemical property (e.g., hydrophobic). Positions were colour-coded green if residues occurred with a higher frequency than any other residues but the group had different physicochemical properties. Positions that are poorly conserved in the consensus sequence were colour-coded yellow and assigned to the amino acid detailed in [53]. (B) Identification of the 22 ankyrin-like repeats (R1-R22) in $\alpha$-LTX-Lh1a. Note the imperfect repeats $\mathrm{R} 1, \mathrm{R} 16$ and R17. The percentage similarity with the consensus sequence is indicated at the right of the sequences. 